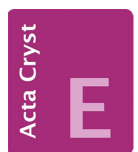

CRYSTALLOGRAPHIC COMMUNICATIONS

ISSN 2056-9890

Received 30 March 2021

Accepted 11 May 2021

Edited by G. Diaz de Delgado, Universidad de Los Andes, Venezuela

Keywords: crystal structure; pyrazolylacetamide; hydrogen bond.

CCDC reference: 2083102

Supporting information: this article has supporting information at journals.iucr.org/e

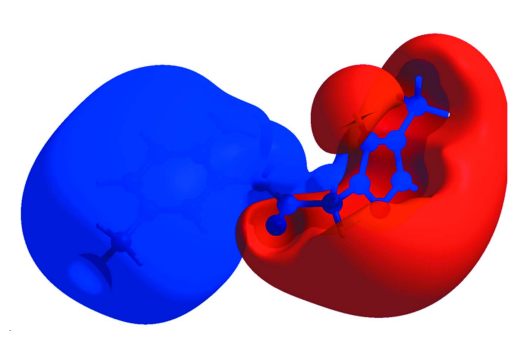

OPEN $\odot$ ACCESS

\section{Crystal structure, Hirshfeld surface analysis and DFT study of $\mathrm{N}$-(2-amino-5-methylphenyl)-2-(5- methyl-1H-pyrazol-3-yl)acetamide}

\author{
Gamal Al Ati, ${ }^{a}$ Karim Chkirate, ${ }^{\text {a Joel T. Mague, }}{ }^{\mathrm{b}}$ Nadeem Abad, ${ }^{\mathrm{c} *}$ Redouane \\ Achour $^{\mathrm{a}}$ and El Mokhtar Essassi ${ }^{\mathrm{a}}$
}

\begin{abstract}
aLaboratory of Heterocyclic Organic Chemistry URAC 21, Pharmacochemistry Competence Center, Av. Ibn Battouta, BP 1014, Faculty of Sciences, Mohammed V University, Rabat, Morocco, ${ }^{\mathbf{b}}$ Department of Chemistry, Tulane University, New Orleans, LA 70118, USA, and ' Department of Biochemistry, Faculty of Education \& Science, Al-Baydha University, Yemen. *Correspondence e-mail: abadnadeem3@gmail.com
\end{abstract}

The title molecule, $\mathrm{C}_{13} \mathrm{H}_{16} \mathrm{~N}_{4} \mathrm{O}$, adopts an angular conformation. In the crystal a layer structure is generated by $\mathrm{N}-\mathrm{H} \cdots \mathrm{O}$ and $\mathrm{N}-\mathrm{H} \cdots \mathrm{N}$ hydrogen bonds together with $\mathrm{C}-\mathrm{H} \cdots \pi(\mathrm{ring})$ interactions. Hirshfeld surface analysis indicates that the most important contributions to the crystal packing are from $\mathrm{H} \cdots \mathrm{H}$ $(53.8 \%), \mathrm{H} \cdots \mathrm{C} / \mathrm{C} \cdots \mathrm{H}(21.7 \%), \mathrm{H} \cdots \mathrm{N} / \mathrm{N} \cdots \mathrm{H}(13.6 \%)$, and $\mathrm{H} \cdots \mathrm{O} / \mathrm{O} \cdots \mathrm{H}$ $(10.8 \%)$ interactions. The optimized structure calculated using density functional theory (DFT) at the B3LYP/ 6-311 G(d,p) level is compared with the experimentally determined structure in the solid state. The calculated HOMO-LUMO energy gap is $5.0452 \mathrm{eV}$.

\section{Chemical context}

Nitrogen-based structures have attracted more attention in recent years because of their interesting properties in structural and inorganic chemistry (Lahmidi et al., 2018; Chkirate et al., 2020a; Taia et al., 2020; Al Ati et al., 2021). The pyrazolylacetamide family is important in medicinal chemistry because of the wide range of pharmacological applications (DeprezPoulain et al., 2011) such as anti-inflammatory (Sunder et al., 2013), antimicrobial and anticancer (Jitender Dev et al., 2017) and as an anti-amoebic agent (Shukla et al., 2020). They also have antioxidant activity (Chkirate et al., 2019a) and have been biologically evaluated (Yan et al., 2021). Given the wide range of therapeutic applications for such compounds, and in a continuation of the work already carried out for the synthesis of compounds resulting from 1,5-benzodiazepine (Chkirate et al., 2001, 2018, 2019b, 2020b, 2021; Idrissi et al., 2021) a similar approach gave the title compound, $N$-(2-amino-5-methylphenyl)-2-(5-methyl-1H-pyrazol-3-yl)acetamide, (I). Besides the synthesis, we also report the molecular and crystal structures along with a Hirshfeld surface analysis and a density functional theory computational calculation carried out at the B3LYP/6-311 G(d,p) level.

\section{Structural commentary}

The $\mathrm{N} 2 / \mathrm{C} 8 / \mathrm{C} 9 / \mathrm{O} 1$ portion of the title molecule is planar (r.m.s. deviation $=0.0013 \AA$ ) with the mean planes of the C1-C6 and $\mathrm{N} 3 / \mathrm{N} 4 / \mathrm{C} 10-\mathrm{C} 12$ rings inclined to the above plane by 86.56 (6) and $72.84(7)^{\circ}$, respectively, giving the molecule an angular 


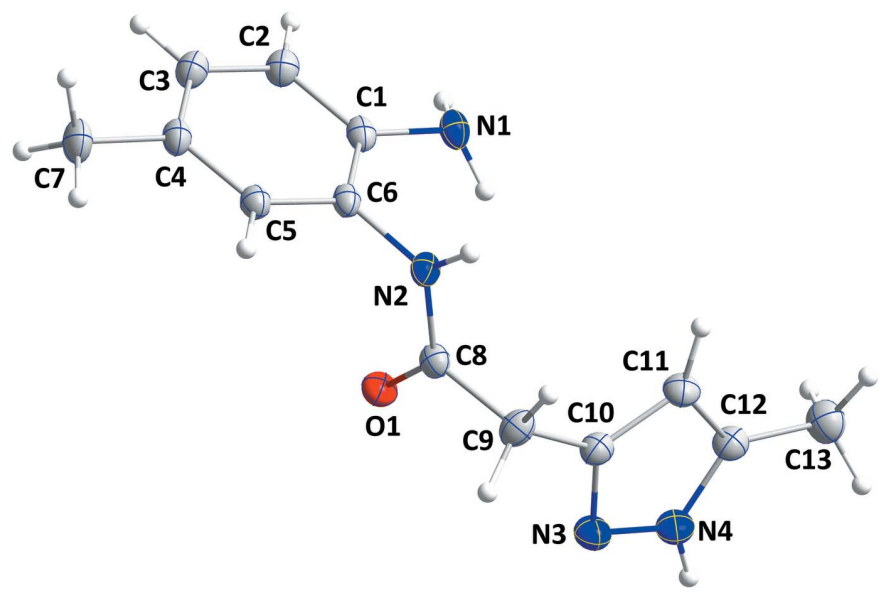

Figure 1

Molecular structure of the title compound with the labelling scheme. The ellipsoids are drawn at the $50 \%$ probability level.

shape (Fig. 1). Bond distances and angles are as expected for the given formulation.<smiles>Cc1ccc(N)c(NC(=O)Cc2cc(C)[nH]n2)c1</smiles>

\section{Supramolecular features}

In the crystal, inversion-related pairs of $\mathrm{N} 1-\mathrm{H} 1 B \cdots \mathrm{O} 1$, and $\mathrm{N} 2-\mathrm{H} 2 A \cdots \mathrm{N} 1$ hydrogen bonds, together with $\mathrm{C} 11-$ $\mathrm{H} 11 \cdots \mathrm{Cg} 2$ interactions (Table 1) form chains of molecules extending along the $a$-axis direction (Fig. 2). The chains are

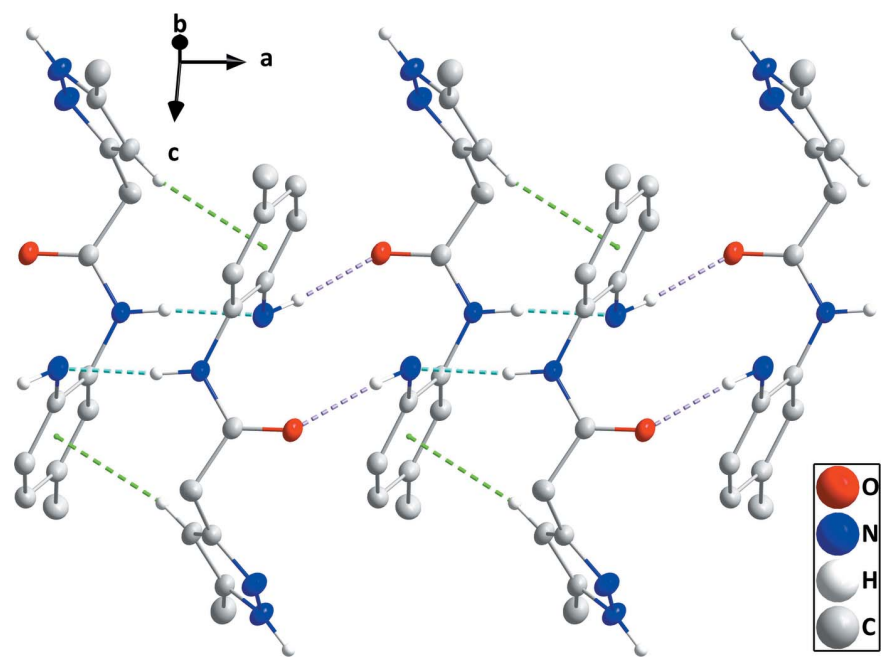

Figure 2

A portion of one chain projected onto (011) with $\mathrm{N}-\mathrm{H} \cdots \mathrm{O}$ and $\mathrm{N}-$ $\mathrm{H} \cdots \mathrm{N}$ hydrogen bonds depicted, respectively, by light-purple and lightblue dashed lines. The $\mathrm{C}-\mathrm{H} \cdots \pi($ ring) interactions are depicted by green dashed lines. Hydrogen atoms not involved in interactions have been omitted for clarity.
Table 1

Hydrogen-bond geometry $\left(\AA,^{\circ}\right)$.

\begin{tabular}{lllll}
\hline$D-\mathrm{H} \cdots A$ & $D-\mathrm{H}$ & $\mathrm{H} \cdots A$ & $D \cdots A$ & $D-\mathrm{H} \cdots A$ \\
\hline $\mathrm{N} 1-\mathrm{H} 1 B \cdots \mathrm{O} 1^{\mathrm{i}}$ & 0.91 & 2.13 & $3.0284(19)$ & 171 \\
$\mathrm{~N} 2-\mathrm{H} 2 A \cdots \mathrm{N} 1^{\mathrm{ii}}$ & 0.91 & 2.14 & $3.0354(17)$ & 170 \\
$\mathrm{C} 2-\mathrm{H} 2 \cdots \mathrm{O} 1^{\mathrm{i}}$ & 0.95 & 2.62 & $3.334(2)$ & 132 \\
$\mathrm{~N} 4-\mathrm{H} 4 \cdots 1^{\mathrm{iii}}$ & $0.91(1)$ & $1.99(1)$ & $2.8625(17)$ & $163(2)$ \\
\hline
\end{tabular}

Symmetry codes: (i) $-x+1,-y+1,-z+1 ; \quad$ (ii) $-x+2,-y+1,-z+1$; (iii) $-x+1, y+\frac{1}{2},-z+\frac{1}{2}$.

connected into layers parallel to (100) by $\mathrm{N} 4-\mathrm{H} 4 \cdots \mathrm{O} 1$ hydrogen bonds (Table 1 and Fig. 3). Intermolecular interactions viewed down the $c$ axis are shown in Fig. 3.

\section{Hirshfeld surface analysis}

The CrystalExplorer program (Turner et al., 2017) was used to investigate and visualize further the intermolecular interactions of (I). The Hirshfeld surface plotted over $d_{\text {norm }}$ in the range -0.6149 to 1.3177 a.u. is shown in Fig. $4 a$. The electrostatic potential calculated using the STO-3G basis set at the Hartree-Fock level of theory and mapped on the Hirshfeld surface over the range \pm 0.05 a.u. clearly shows the positions of close intermolecular contacts in the compound (Fig. $4 b$ ). The positive electrostatic potential (blue region) over the surface indicates hydrogen-donor potential, whereas the hydrogenbond acceptors are represented by negative electrostatic potential (red region). The shape-index (Fig. 5) generated in the range -1 to $1 \AA$ reveals that there are no significant $\pi-\pi$ interactions, normally indicated by adjacent red and blue triangles.

The overall two-dimensional fingerprint plot (McKinnon et $a l ., 2007)$ is shown in Fig. $6 a$, while those delineated into $\mathrm{H} \cdots \mathrm{H}, \quad \mathrm{H} \cdots \mathrm{C} / \mathrm{C} \cdots \mathrm{H}, \quad \mathrm{H} \cdots \mathrm{N} / \mathrm{N} \cdots \mathrm{H}$ and $\mathrm{H} \cdots \mathrm{O} / \mathrm{O} \cdots \mathrm{H}$ contacts are illustrated in Fig. $6 b-e$, respectively, together with their relative contributions to the Hirshfeld surface (HS). The most important interaction is $\mathrm{H} \cdots \mathrm{H}$, contributing $53.8 \%$ to the overall crystal packing, which is reflected in Fig. $6 b$ as widely scattered points of high density due to the large hydrogen content of the molecule, with the tip at $d_{\mathrm{e}}=d_{\mathrm{i}}=$ $1.18 \AA$. In the presence of $\mathrm{C}-\mathrm{H}$ interactions, the pair of characteristic wings in the fingerprint plot delineated into $\mathrm{H} \cdots \mathrm{C} / \mathrm{C} \cdots \mathrm{H}$ contacts $(21.7 \%$ contribution to the HS), Fig. $6 c$,

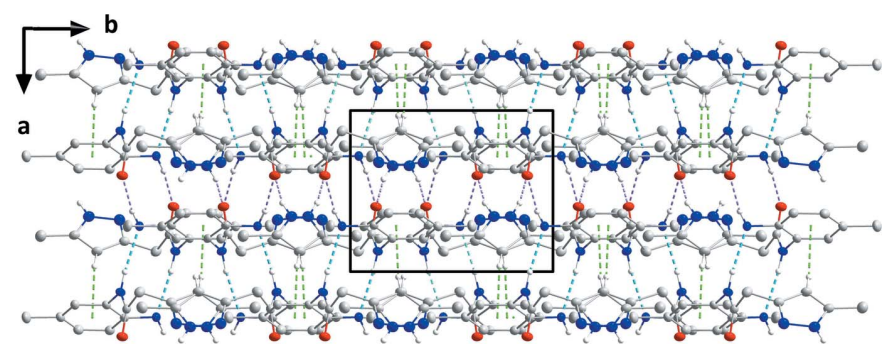

Figure 3

Packing arrangement viewed along the $c$-axis direction of the main isomer with intermolecular interactions shown as in Fig. 2. 

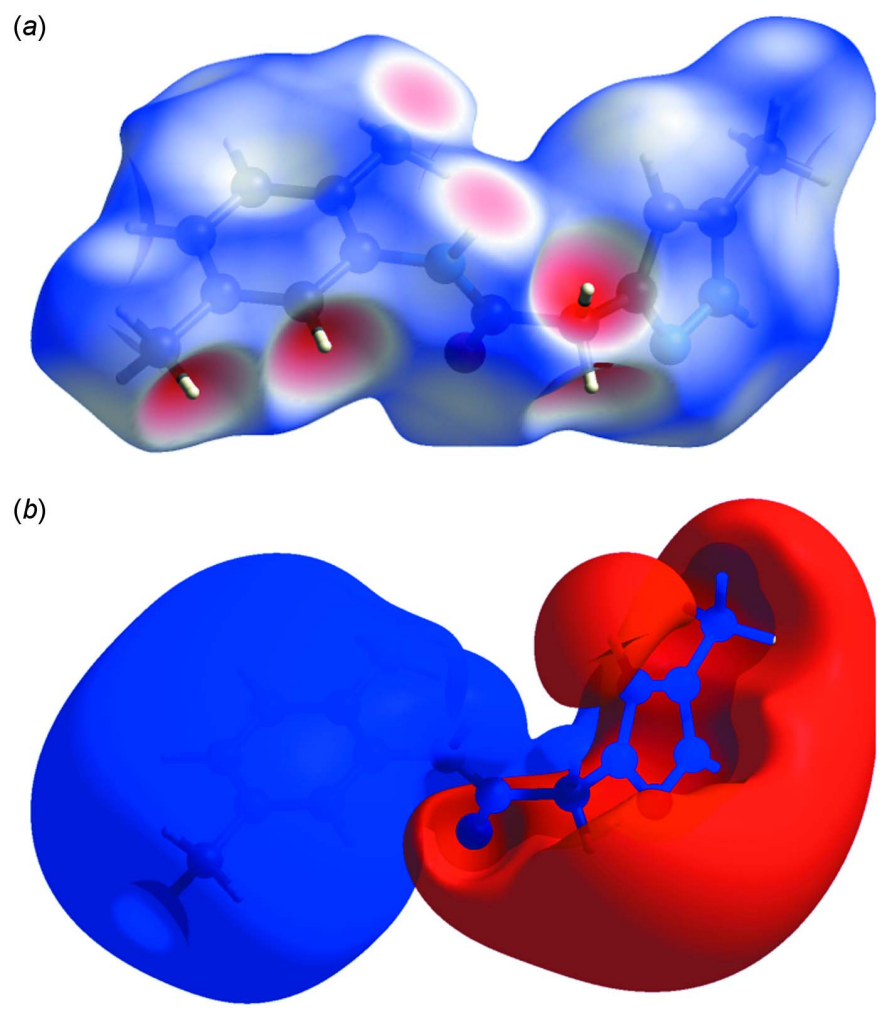

Figure 4

(a) View of the three-dimensional Hirshfeld surface of the title compound, plotted over $d_{\text {norm }}$ in the range of -0.6149 to 1.3177 a.u. (b) View of the three-dimensional Hirshfeld surface of the title compound plotted over the electrostatic potential energy in the range -0.0500 to 0.0500 a.u. using the STO-3 G basis set at the Hartree-Fock level of theory.

has the tips at $d_{\mathrm{e}}+d_{\mathrm{i}}=2.76 \AA$. The pair of scattered points of spikes in the fingerprint plot delineated into $\mathrm{H} \cdots \mathrm{N} / \mathrm{N} \cdots \mathrm{H}$, Fig. $6 d(13.6 \%)$, have the tips at $d_{\mathrm{e}}+d_{\mathrm{i}}=2.01 \AA$. Finally, the $\mathrm{H} \cdots \mathrm{O} / \mathrm{O} \cdots \mathrm{H}$ contacts, Fig. $6 e$, make only a $10.8 \%$ contribution to the HS and have a low-density distribution of points.

\section{Density functional theory calculations}

The structure in the gas phase of the title compound was optimized by means of density functional theory. The density

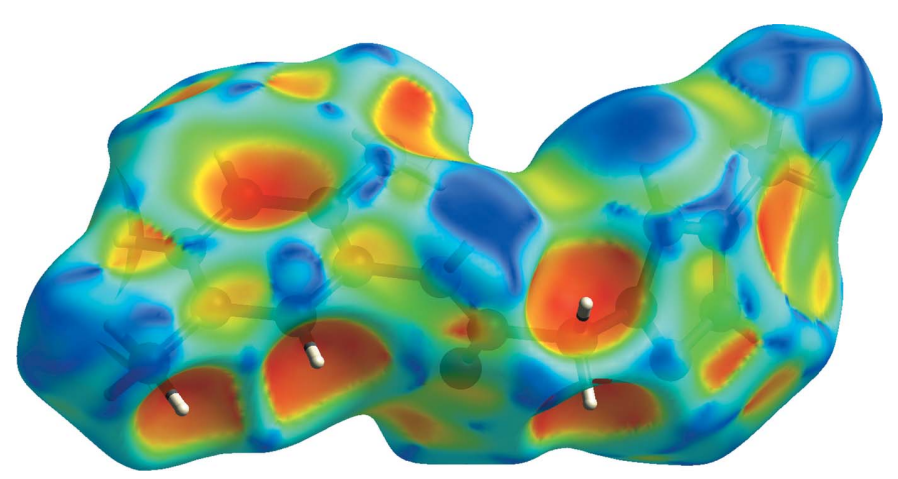

Figure 5

Hirshfeld surface of the title compound plotted over shape-index.
Table 2

Comparison of selected (X-ray and density functional theory) bond lengths and angles $\left(\AA{ }^{\circ}\right)$.

\begin{tabular}{lll}
\hline & X-ray & B3LYP/6-311G(d,p) \\
\hline N1-C1 & $1.4112(17)$ & 1.4114 \\
N2-C6 & $1.4347(17)$ & 1.4139 \\
N2-C8 & $1.3471(17)$ & 1.3692 \\
O1-C8 & $1.2376(16)$ & 1.2179 \\
N3-C10 & $1.3425(18)$ & 1.3316 \\
N3-N4 & $1.3635(19)$ & 1.3524 \\
N4-C12 & $1.3534(19)$ & 1.3598 \\
C8-C9 & $1.5119(18)$ & 1.5409 \\
C9-C10 & $1.496(2)$ & 1.5007 \\
C2-C1-N1 & & \\
C6-C1-N1 & $121.12(12)$ & 122.0542 \\
C1-C6-N2 & $120.68(12)$ & 119.3119 \\
C5-C6-N2 & $119.89(11)$ & 116.726 \\
O1-C8-N2 & $119.46(12)$ & 123.4969 \\
N2-C8-C9 & $122.20(12)$ & 125.0222 \\
O1-C8-C9 & $116.22(11)$ & 114.6561 \\
N3-C10-C9 & $121.58(12)$ & 120.2798 \\
N3-C10-C11 & $119.95(13)$ & 120.7841 \\
C10-N3-N4 & $111.17(12)$ & 110.8968 \\
C12-N4-N3 & $104.22(12)$ & 104.754 \\
N4-C12-C11 & $112.76(12)$ & 113.2928 \\
N4-C12-C13 & $106.48(13)$ & 105.3557 \\
\hline
\end{tabular}

Table 3

Calculated energies.

\begin{tabular}{lr}
\hline Molecular Energy & Compound $(\mathrm{I})$ \\
\hline Total Energy $T E(\mathrm{eV})$ & -21754.8403 \\
$E_{\mathrm{HOMO}}(\mathrm{eV})$ & -5.3130 \\
$E_{\mathrm{LUMO}}(\mathrm{eV})$ & -0.2678 \\
$\mathrm{Gap}, \Delta E(\mathrm{eV})$ & 5.0452 \\
Dipole moment, $\mu($ Debye $)$ & 6.7706 \\
Ionization potential, $I(\mathrm{eV})$ & 5.3130 \\
Electron affinity, $A$ & 0.2678 \\
Electronegativity, $\chi$ & 2.7904 \\
Hardness, $\eta$ & 2.5226 \\
Electrophilicity, index $\omega$ & 1.5433 \\
Softness, $\sigma$ & 0.3964 \\
Fraction of electron transferred, $\Delta N$ & 0.8344 \\
\hline
\end{tabular}

functional theory calculation was performed by the hybrid B3LYP method and the 6-311 G(d,p) basis-set, which is based on Becke's model (Becke, 1993) and considers a mixture of the exact (Hartree-Fock) and density functional theory exchange utilizing the $\mathrm{B} 3$ functional, together with the LYP correlation functional (Lee et al., 1988). After obtaining the converged geometry, the harmonic vibrational frequencies were calculated at the same theoretical level to confirm that the number of imaginary frequencies is zero for the stationary point. Both the geometry optimization and harmonic vibrational frequency analysis of the title compound were done with the Gaussian 09 program (Frisch et al., 2009). Theoretical and experimental results related to bond lengths and angles are in good agreement and are summarized in Table 2. Calculated numerical values for the title compound including electronegativity $(\chi)$, hardness $(\eta)$, ionization potential $(I)$, dipole moment $(\mu)$, electron affinity $(A)$, electrophilicity $(\omega)$ and softness $(\sigma)$ are collated in Table 3 . The electron transition from the highest occupied molecular orbital (HOMO) to the 

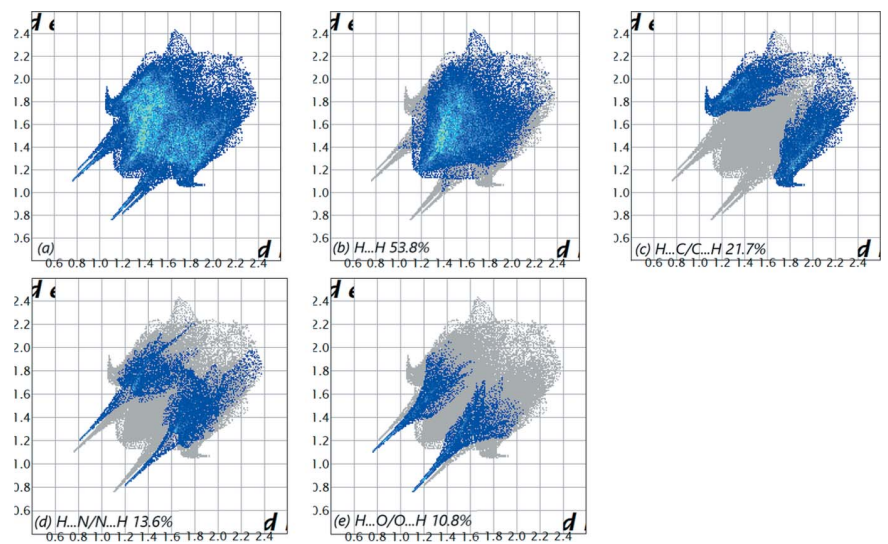

Figure 6

The full two-dimensional fingerprint plots for the title compound, showing (a) all interactions, and those delineated into $(b) \mathrm{H} \cdots \mathrm{H},(c)$ $\mathrm{H} \cdots \mathrm{C} / \mathrm{C} \cdots \mathrm{H},(d) \mathrm{H} \cdots \mathrm{N} / \mathrm{N} \cdots \mathrm{H}$ and $(e) \mathrm{H} \cdots \mathrm{O} / \mathrm{O} \cdots \mathrm{H}$ interactions. The $d_{\mathrm{i}}$ and $d_{\mathrm{e}}$ values are the closest internal and external distances (in $\AA$ ) from given points on the Hirshfeld surface.

lowest unoccupied molecular orbital (LUMO) energy level is shown in Fig. 7. The HOMO and LUMO are localized in the plane extending over the whole $N$-(2-amino-5-methylphenyl)2-(5-methyl-1H-pyrazol-3-yl)acetamide system. The energy
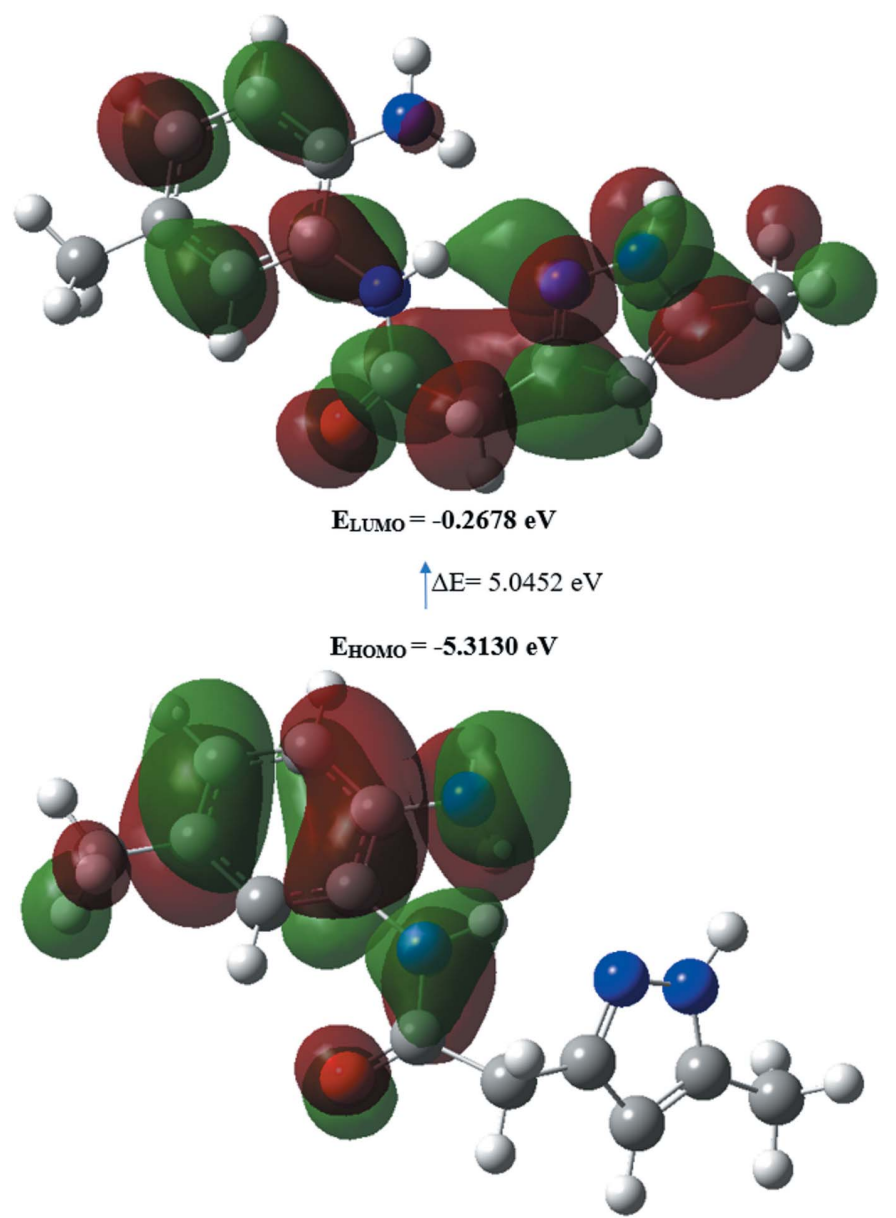

Figure 7

The energy band gap of $\mathrm{N}$-(2-amino-5-methylphenyl)-2-(5-methyl-1 $\mathrm{H}$ pyrazol-3-yl)acetamide. band gap $\left[\triangle E=E_{L U M O}-E_{\text {Номо }}\right]$ of the molecule is $5.0452 \mathrm{eV}$, and the frontier molecular orbital energies, $E_{\text {Номо }}$ and $E_{L U M O}$, are -5.3130 and $-0.2678 \mathrm{eV}$, respectively.

\section{Database survey}

A search of the Cambridge Structural Database (CSD version 5.40, updated March 2020; Groom et al., 2016) with the 2-(5methyl-1H-pyrazol-3-yl)acetamide fragment yielded multiple matches. Of these, two had an $N$-(2-aminophenyl) substituent comparable to (I) and they are shown in Fig. 8. The first compound (II) (refcode XITFUE; Chkirate et al., 2019c) carries $\quad N-(2-\{[(4-m e t h y l p h e n y l) m e t h y l i d e n e] a m i n o\}$ phenyl $)$ on nitrogen 2. The second one (III) (refcode YODZEZ; Chkirate et al., 2019a) carries $N$-(2-aminophenyl) on nitrogen 2. The pyrazole ring $(\mathrm{N} 3 / \mathrm{N} 4 / \mathrm{C} 10-\mathrm{C} 12)$ in XITFUE is inclined to the $\mathrm{C} 1-\mathrm{C} 6$ benzene ring by $70.83(8)^{\circ}$. In YODZEZ, the dihedral angle between the mean planes of the 2-aminophenyl and pyrazolyl rings is $65.63(8)^{\circ}$. In (I), the $\mathrm{N} 2 / \mathrm{C} 8 / \mathrm{C} 9 / \mathrm{O} 1$ fragment is planar (r.m.s. deviation $=0.0013 \AA$ ) with the mean planes of the $\mathrm{C} 1-\mathrm{C} 6$ and $\mathrm{N} 3 / \mathrm{N} 4 / \mathrm{C} 10-\mathrm{C} 12$ rings inclined to the above plane by 86.56 (6) and $72.84(7)^{\circ}$, respectively, which is approximately the same as in XITFUE, but less tilted than in YODZEZ.

\section{Synthesis and crystallization}

$2 \mathrm{~g}$ (9.3 mmol) of (Z)-4-(2-oxopropylidene)-1,5-benzodiazepin-2-one and a stoichiometric amount of hydrazine were refluxed in ethanol $(40 \mathrm{~mL})$ for $2 \mathrm{~h}$. After concentration of the solvent volume to $20 \mathrm{~mL}$, the solution was allowed to stand; the precipitate formed was filtered off and then recrystallized in ethanol. Single crystals were obtained after recrystallization from methanol in the presence of $\mathrm{MnCl}_{2} \cdot 4 \mathrm{H}_{2} \mathrm{O}$, which was left at room temperature for $72 \mathrm{~h}$. Yield: $70 \%$.

\section{Refinement}

Crystal data, data collection and structure refinement details are summarized in Table $4 . \mathrm{H}$ atoms were included as riding contributions in idealized positions $(\mathrm{N}-\mathrm{H}=0.88-0.91 \AA, \mathrm{C}-$ $\mathrm{H}=0.95-0.99 \AA$ ) with isotropic displacement parameters $1.2-$

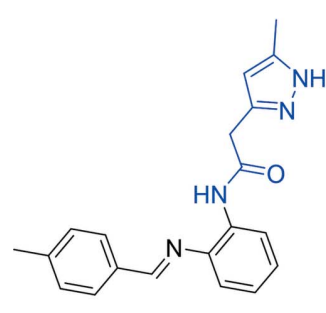

II

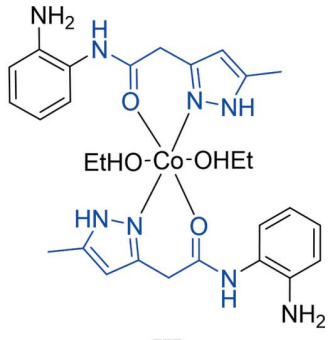

III
Figure 8

Structures similar to (I): (II) (CSD refcode XITFUE) and (III) (CSD refcode YODZEZ) obtained in the database search. The search fragment is indicated in blue. 
Table 4

Experimental details.

\begin{tabular}{ll}
\hline Crystal data & \\
Chemical formula & $\mathrm{C}_{13} \mathrm{H}_{16} \mathrm{~N}_{4} \mathrm{O}$ \\
$M_{\mathrm{r}}$ & 244.30 \\
Crystal system, space group & Monoclinic, $P 2_{1} / c$ \\
Temperature $(\mathrm{K})$ & 150 \\
$a, b, c(\AA)$ & $7.1271(3), 8.9295(3), 19.2508(7)$ \\
$\beta\left({ }^{\circ}\right.$ & $94.683(1)$ \\
$V\left(\AA^{3}\right)$ & $1221.06(8)$ \\
$Z$ & 4 \\
Radiation type & Mo $K \alpha$ \\
$\mu\left(\mathrm{mm}^{-1}\right)$ & 0.09 \\
Crystal size (mm) & $0.37 \times 0.26 \times 0.16$ \\
& \\
Data collection & Bruker D8 QUEST PHOTON 3 \\
Diffractometer & diffractometer \\
& Numerical $(S A D A B S ;$ Krause $e t$ \\
Absorption correction & al., 2015$)$ \\
& $0.93,0.99$ \\
$T_{\text {min }}, T_{\text {max }}$ & $66426,5120,4794$ \\
No. of measured, independent and & \\
$\quad$ observed $[I>2 \sigma(I)]$ reflections & 0.028 \\
$R_{\text {int }}$ & 0.794 \\
sin $\theta / \lambda)_{\text {max }}\left(\AA^{-1}\right)$ & \\
Refinement & \\
$R\left[F^{2}>2 \sigma\left(F^{2}\right)\right], w R\left(F^{2}\right), S$ & $0.067,0.164,1.23$ \\
No. of reflections & 5120 \\
No. of parameters & 212 \\
No. of restraints & 32 \\
H-atom treatment & H atoms treated by a mixture of \\
& independent and constrained \\
$\Delta \rho_{\text {max }}, \Delta \rho_{\text {min }}\left(\mathrm{e} \AA^{-3}\right)$ & refinement \\
\hline & $0.44,-0.33$ \\
\hline
\end{tabular}

Computer programs: APEX3 and SAINT (Bruker, 2020), SHELXT (Sheldrick, 2015a), SHELXL2018/1 (Sheldrick, 2015b), DIAMOND (Brandenburg \& Putz, 2012) and SHELXTL (Sheldrick, 2008).

1.5 times those of the attached atoms. Residual density observed after the initial refinement converged was identified as an isomer of the primary molecule having the $\mathrm{C} 7$ methyl group attached to $\mathrm{C} 3$ instead of to $\mathrm{C} 4$ and with a refined occupancy of $5 \%$. The final model was generated with a combination of rigid group and restrained refinement to make the minor component have a comparable geometry to that of the major component.

\section{Acknowledgements}

JTM thanks Tulane University for support of the Tulane Crystallography Laboratory. Author contribution are as follows. Conceptualization, GAA and $\mathrm{KC}$; methodology, GAA; investigation, $\mathrm{KC}$ and $\mathrm{NA}$; theoretical calculations, $\mathrm{KC}$; writing (original draft) $\mathrm{KC}$; writing (review and editing of the manuscript) NA; supervision, KC, EME and RA; crystalstructure determination and validation, JTM.

\section{References}

Al Ati, G., Chkirate, K., Mashrai, A., Mague, J. T., Ramli, Y., Achour, R. \& Essassi, E. M. (2021). Acta Cryst. E77, 18-22.

Becke, A. D. (1993). J. Chem. Phys. 98, 5648-5652.

Brandenburg, K. \& Putz, H. (2012). DIAMOND. Crystal Impact GbR, Bonn, Germany.

Bruker (2020). APEX3 and SAINT. Bruker AXS Inc., Madison, Wisconsin, USA.
Chkirate, K., Azgaou, K., Elmsellem, H., El Ibrahimi, B., Sebbar, N. K., Anouar, E. H., Benmessaoud, M., El Hajjaji, S. \& Essassi, E. M. (2021). J. Mol. Liq. 321, 114750.

Chkirate, K., Fettach, S., El Hafi, M., Karrouchi, K., Elotmani, B., Mague, J. T., Radi, S., Faouzi, M. E. A., Adarsh, N. N., Essassi, E. M. \& Garcia, Y. (2020a). J. Inorg. Biochem. 208, 21-28.

Chkirate, K., Fettach, S., Karrouchi, K., Sebbar, N. K., Essassi, E. M., Mague, J. T., Radi, S., Faouzi, M. E. A., Adarsh, N. N. \& Garcia, Y. (2019a). J. Inorg. Biochem. 191, 21-28.

Chkirate, K., Kansiz, S., Karrouchi, K., Mague, J. T., Dege, N. \& Essassi, E. M. (2019b). Acta Cryst. E75, 33-37.

Chkirate, K., Kansiz, S., Karrouchi, K., Mague, J. T., Dege, N. \& Essassi, E. M. (2019c). Acta Cryst. E75, 154-158.

Chkirate, K., Karrouchi, K., Dege, N., Sebbar, N. K., Ejjoummany, A., Radi, S., Adarsh, N. N., Talbaoui, A., Ferbinteanu, M., Essassi, E. M. \& Garcia, Y. (2020b). New J. Chem. 44, 2210-2221.

Chkirate, K., Regragui, R., Essassi, E. M. \& Pierrot, M. (2001). Z. Kristallogr. New Cryst. Struct. 216, 635-636.

Chkirate, K., Sebbar, N. K., Hökelek, T., Krishnan, D., Mague, J. T. \& Essassi, E. M. (2018). Acta Cryst. E74, 1669-1673.

Deprez-Poulain, R., Cousaert, N., Toto, P., Willand, N. \& Deprez, B. (2011). Eur. J. Med. Chem. 46, 3867-3876.

Frisch, M. J., Trucks, G. W., Schlegel, H. B., Scuseria, G. E., Robb, M. A., Cheeseman, J. R., Scalmani, G., Barone, V., Mennucci, B., Petersson, G. A., Nakatsuji, H., Caricato, M., Li, X., Hratchian, H. P., Izmaylov, A. F., Bloino, J., Zheng, G., Sonnenberg, J. L., Hada, M., Ehara, M., Toyota, K., Fukuda, R., Hasegawa, J., Ishida, M., Nakajima, T., Honda, Y., Kitao, O., Nakai, H., Vreven, T., Montgomery, J. A. Jr, Peralta, J. E., Ogliaro, F., Bearpark, M., Heyd, J. J., Brothers, E., Kudin, K. N., Staroverov, V. N., Kobayashi, R., Normand, J., Raghavachari, K., Rendell, A., Burant, J. C., Iyengar, S. S., Tomasi, J., Cossi, M., Rega, N., Millam, J. M., Klene, M., Knox, J. E., Cross, J. B., Bakken, V., Adamo, C., Jaramillo, J., Gomperts, R., Stratmann, R. E., Yazyev, O., Austin, A. J., Cammi, R., Pomelli, C., Ochterski, J. W., Martin, R. L., Morokuma, K., Zakrzewski, V. G., Voth, G. A., Salvador, P., Dannenberg, J. J., Dapprich, S., Daniels, A. D., Farkas, O., Foresman, J. B., Ortiz, J. V., Cioslowski, J. \& Fox, D. J. (2009). Gaussian 09. Revision A.02. Gaussian Inc, Wallingford, CT, USA.

Groom, C. R., Bruno, I. J., Lightfoot, M. P. \& Ward, S. C. (2016). Acta Cryst. B72, 171-179.

Idrissi, A., Chkirate, K., Abad, N., Djerrari, B., Achour, R., Essassi, E. M. \& Van Meervelt, L. (2021). Acta Cryst. E77, 396-401.

Krause, L., Herbst-Irmer, R., Sheldrick, G. M. \& Stalke, D. (2015). J. Appl. Cryst. 48, 3-10.

Lahmidi, S., Sebbar, N. K., Hökelek, T., Chkirate, K., Mague, J. T. \& Essassi, E. M. (2018). Acta Cryst. E74, 1833-1837.

Lee, C., Yang, W. \& Parr, R. G. (1988). Phys. Rev. B, 37, 785-789.

McKinnon, J. J., Jayatilaka, D. \& Spackman, M. A. (2007). Chem. Commun. 3814-3816.

Jitender Dev, G., Poornachandra, Y., Ratnakar Reddy, K., Naresh Kumar, R., Ravikumar, N., Krishna Swaroop, D., Ranjithreddy, P., Shravan Kumar, G., Nanubolu, J. B., Ganesh Kumar, C. \& Narsaiah, B. (2017). Eur. J. Med. Chem. 130, 223-239.

Sheldrick, G. M. (2008). Acta Cryst. A64, 112-122.

Sheldrick, G. M. (2015a). Acta Cryst. A71, 3-8.

Sheldrick, G. M. (2015b). Acta Cryst. C71, 3-8.

Shukla, B. K. \& Yadava, U. (2020). Heliyon, 6, e04176.

Sunder, K. S. \& Maleraju, J. (2013). Drug Invent. Today, 5, 288295.

Taia, A., Essaber, M., Aatif, A., Chkirate, K., Hökelek, T., Mague, J. T. \& Sebbar, N. K. (2020). Acta Cryst. E76, 962-966.

Turner, M. J., McKinnon, J. J., Wolff, S. K., Grimwood, D. J., Spackman, P. R., Jayatilaka, D. \& Spackman, M. A. (2017). CrystalExplorer17. The University of Western Australia.

Yan, W., Zhang, L., Lv, F., Moccia, M., Carlomagno, F., Landry, C., Santoro, M., Gosselet, F., Frett, B. \& Li, H. (2021). Eur. J. Med. Chem. 216, 113265. 


\section{supporting information}

Acta Cryst. (2021). E77, 638-642［https://doi.org/10.1107/S205698902100503X]

Crystal structure, Hirshfeld surface analysis and DFT study of $\mathrm{N}$-(2-amino-5methylphenyl)-2-(5-methyl-1 $H$-pyrazol-3-yl)acetamide

\section{Gamal Al Ati, Karim Chkirate, Joel T. Mague, Nadeem Abad, Redouane Achour and El} Mokhtar Essassi

\section{Computing details}

Data collection: APEX3 (Bruker, 2020); cell refinement: SAINT (Bruker, 2020); data reduction: SAINT (Bruker, 2020); program(s) used to solve structure: SHELXT (Sheldrick, 2015a); program(s) used to refine structure: SHELXL2018/1 (Sheldrick, 2015b); molecular graphics: DIAMOND (Brandenburg \& Putz, 2012); software used to prepare material for publication: SHELXTL (Sheldrick, 2008).

N-(2-Amino-5-methylphenyl)-2-(5-methyl-1H-pyrazol-3-yl)acetamide

Crystal data

$\mathrm{C}_{13} \mathrm{H}_{16} \mathrm{~N}_{4} \mathrm{O}$

$M_{r}=244.30$

Monoclinic, $P 2_{1} / c$

$a=7.1271(3) \AA$

$b=8.9295(3) \AA$

$c=19.2508(7) \AA$

$\beta=94.683(1)^{\circ}$

$V=1221.06(8) \AA^{3}$

$Z=4$

\section{Data collection}

Bruker D8 QUEST PHOTON 3 diffractometer

Radiation source: fine-focus sealed tube Graphite monochromator

Detector resolution: 7.3910 pixels $\mathrm{mm}^{-1}$ $\varphi$ and $\omega$ scans

Absorption correction: numerical (SADABS; Krause et al., 2015)

$T_{\min }=0.93, T_{\max }=0.99$

\section{Refinement}

Refinement on $F^{2}$

Least-squares matrix: full

$R\left[F^{2}>2 \sigma\left(F^{2}\right)\right]=0.067$

$w R\left(F^{2}\right)=0.164$

$S=1.23$

5120 reflections

212 parameters

32 restraints
$F(000)=520$

$D_{\mathrm{x}}=1.329 \mathrm{Mg} \mathrm{m}^{-3}$

Mo $K \alpha$ radiation, $\lambda=0.71073 \AA$

Cell parameters from 9878 reflections

$\theta=2.5-34.4^{\circ}$

$\mu=0.09 \mathrm{~mm}^{-1}$

$T=150 \mathrm{~K}$

Parallelepiped, colourless

$0.37 \times 0.26 \times 0.16 \mathrm{~mm}$

66426 measured reflections

5120 independent reflections

4794 reflections with $I>2 \sigma(I)$

$R_{\text {int }}=0.028$

$\theta_{\text {max }}=34.4^{\circ}, \theta_{\min }=2.5^{\circ}$

$h=-11 \rightarrow 11$

$k=-14 \rightarrow 14$

$l=-30 \rightarrow 30$

Primary atom site location: dual

Secondary atom site location: difference Fourier map

Hydrogen site location: mixed

$\mathrm{H}$ atoms treated by a mixture of independent and constrained refinement

$w=1 /\left[\sigma^{2}\left(F_{\mathrm{o}}^{2}\right)+(0.0465 P)^{2}+0.8885 P\right]$ where $P=\left(F_{\mathrm{o}}^{2}+2 F_{\mathrm{c}}^{2}\right) / 3$ 
$(\Delta / \sigma)_{\max }=0.007$

$\Delta \rho_{\max }=0.44$ e $\AA^{-3}$

\section{Special details}

Experimental. The diffraction data were obtained from 9 sets of frames, each of width $0.5^{\circ}$ in $\omega$ or $\varphi$, collected with scan parameters determined by the "strategy" routine in APEX3. The scan time was $15 \mathrm{sec} /$ frame.

Geometry. All esds (except the esd in the dihedral angle between two 1.s. planes) are estimated using the full covariance matrix. The cell esds are taken into account individually in the estimation of esds in distances, angles and torsion angles; correlations between esds in cell parameters are only used when they are defined by crystal symmetry. An approximate (isotropic) treatment of cell esds is used for estimating esds involving l.s. planes.

Refinement. Refinement of $\mathrm{F}^{2}$ against ALL reflections. The weighted R-factor $\mathrm{wR}$ and goodness of fit $\mathrm{S}$ are based on $\mathrm{F}^{2}$, conventional $\mathrm{R}$-factors $\mathrm{R}$ are based on $\mathrm{F}$, with $\mathrm{F}$ set to zero for negative $\mathrm{F}^{2}$. The threshold expression of $\mathrm{F}^{2}>2 \operatorname{sigma}\left(\mathrm{F}^{2}\right)$ is used only for calculating R-factors(gt) etc. and is not relevant to the choice of reflections for refinement. R-factors based on $\mathrm{F}^{2}$ are statistically about twice as large as those based on F, and R- factors based on ALL data will be even larger. H-atoms were included as riding contributions in idealized positions with isotropic displacement parameters 1.2 1.5 times those of the attached atoms. Residual density observed after the initial refinement converged was identified as an isomer of the primary molecule having the $\mathrm{C} 7$ methyl group attached to $\mathrm{C} 3$ instead of to $\mathrm{C} 4$ and with a refined occupancy of 5\%. The final model was generated with a combination of rigid group and restrained refinement to make the minor component have a comparable geometry to that of the major component.

Fractional atomic coordinates and isotropic or equivalent isotropic displacement parameters $\left(\AA^{2}\right)$

\begin{tabular}{|c|c|c|c|c|c|}
\hline & $x$ & $y$ & $z$ & $U_{\text {iso }} * / U_{\text {eq }}$ & Occ. $(<1)$ \\
\hline O1 & $0.59624(16)$ & $0.37274(17)$ & $0.37033(6)$ & $0.0232(2)$ & $0.9480(17)$ \\
\hline N1 & $0.71759(18)$ & $0.54183(13)$ & $0.54033(7)$ & $0.0217(2)$ & $0.9480(17)$ \\
\hline $\mathrm{H} 1 \mathrm{~A}$ & 0.705685 & 0.575800 & 0.495619 & $0.032 *$ & $0.9480(17)$ \\
\hline H1B & 0.625376 & 0.578103 & 0.565705 & $0.032 *$ & $0.9480(17)$ \\
\hline $\mathrm{N} 2$ & $0.87261(16)$ & $0.36438(14)$ & $0.43708(6)$ & $0.0194(2)$ & $0.9480(17)$ \\
\hline $\mathrm{H} 2 \mathrm{~A}$ & 0.997675 & 0.386010 & 0.438829 & $0.029 *$ & $0.9480(17)$ \\
\hline $\mathrm{O} 1 \mathrm{~A}$ & $0.611(2)$ & $0.392(4)$ & $0.3551(15)$ & $0.0232(2)$ & $0.0520(17)$ \\
\hline $\mathrm{N} 1 \mathrm{~A}$ & $0.726(2)$ & $0.5270(12)$ & $0.5246(10)$ & $0.0217(2)$ & $0.0520(17)$ \\
\hline $\mathrm{H} 1 \mathrm{C}$ & 0.714407 & 0.560992 & 0.479901 & $0.032 *$ & $0.0520(17)$ \\
\hline H1D & 0.634098 & 0.563294 & 0.549991 & $0.032 *$ & $0.0520(17)$ \\
\hline $\mathrm{N} 2 \mathrm{~A}$ & $0.892(3)$ & $0.379(3)$ & $0.4164(9)$ & $0.0194(2)$ & $0.0520(17)$ \\
\hline $\mathrm{H} 2 \mathrm{C}$ & 1.010632 & 0.408561 & 0.419618 & $0.023^{*}$ & $0.0520(17)$ \\
\hline $\mathrm{C} 1$ & $0.71306(17)$ & $0.38417(14)$ & $0.54490(7)$ & $0.0183(2)$ & $0.9480(17)$ \\
\hline $\mathrm{C} 2$ & 0.63639 (19) & $0.31229(16)$ & $0.60084(8)$ & $0.0211(2)$ & 0.9480 (17) \\
\hline $\mathrm{H} 2$ & 0.581442 & 0.370326 & 0.635231 & $0.025 *$ & $0.9480(17)$ \\
\hline $\mathrm{C} 3$ & 0.63985 (19) & $0.15736(16)$ & $0.60656(8)$ & $0.0217(2)$ & $0.9480(17)$ \\
\hline $\mathrm{H} 3$ & 0.588038 & 0.111015 & 0.645085 & $0.026^{*}$ & $0.9480(17)$ \\
\hline $\mathrm{C} 4$ & $0.71811(19)$ & $0.06826(15)$ & $0.55670(7)$ & $0.0201(2)$ & $0.9480(17)$ \\
\hline $\mathrm{C} 5$ & $0.79467(18)$ & $0.13954(15)$ & $0.50122(7)$ & $0.0190(2)$ & $0.9480(17)$ \\
\hline H5 & 0.848761 & 0.081079 & 0.466773 & $0.023^{*}$ & $0.9480(17)$ \\
\hline C6 & $0.79337(17)$ & $0.29531(14)$ & $0.49529(7)$ & $0.0173(2)$ & $0.9480(17)$ \\
\hline $\mathrm{C} 7$ & $0.7163(2)$ & $-0.10021(16)$ & $0.56184(9)$ & 0.0267 & $0.9480(17)$ \\
\hline $\mathrm{H} 7 \mathrm{~A}$ & 0.762818 & -0.143428 & 0.519718 & $0.040 *$ & $0.9480(17)$ \\
\hline H7B & 0.587355 & -0.134775 & 0.566403 & $0.040 *$ & $0.9480(17)$ \\
\hline $\mathrm{H} 7 \mathrm{C}$ & 0.797458 & -0.131927 & 0.602734 & $0.040 *$ & $0.9480(17)$ \\
\hline $\mathrm{C} 8$ & $0.76818(18)$ & $0.39484(15)$ & $0.37725(7)$ & $0.0181(2)$ & $0.9480(17)$ \\
\hline C9 & $0.87268(19)$ & $0.45874(17)$ & $0.31874(7)$ & 0.0233 & $0.9480(17)$ \\
\hline
\end{tabular}




\begin{tabular}{|c|c|c|c|c|c|}
\hline H9A & 1.009431 & 0.458403 & 0.332792 & $0.028^{*}$ & $0.9480(17)$ \\
\hline H9B & 0.850612 & 0.394239 & 0.277043 & $0.028^{*}$ & $0.9480(17)$ \\
\hline $\mathrm{C} 1 \mathrm{~A}$ & $0.7392(15)$ & $0.3693(12)$ & $0.5253(6)$ & $0.0183(2)$ & $0.0520(17)$ \\
\hline $\mathrm{C} 2 \mathrm{~A}$ & $0.664(2)$ & $0.2879(16)$ & $0.5780(7)$ & $0.0211(2)$ & $0.0520(17)$ \\
\hline $\mathrm{H} 2 \mathrm{~B}$ & 0.604271 & 0.338398 & 0.613539 & $0.025^{*}$ & $0.0520(17)$ \\
\hline $\mathrm{C} 3 \mathrm{~A}$ & $0.677(2)$ & $0.1325(16)$ & $0.5786(8)$ & $0.0217(2)$ & $0.0520(17)$ \\
\hline $\mathrm{C} 4 \mathrm{~A}$ & $0.764(2)$ & $0.0586(12)$ & $0.5266(9)$ & $0.0201(2)$ & $0.0520(17)$ \\
\hline $\mathrm{H} 4 \mathrm{~A}$ & 0.773122 & -0.047555 & 0.527024 & $0.024 *$ & $0.0520(17)$ \\
\hline $\mathrm{C} 5 \mathrm{~A}$ & $0.839(2)$ & $0.1400(14)$ & $0.4739(8)$ & $0.0190(2)$ & $0.0520(17)$ \\
\hline $\mathrm{H} 5 \mathrm{~A}$ & 0.899373 & 0.089494 & 0.438300 & $0.023^{*}$ & $0.0520(17)$ \\
\hline C6A & $0.827(2)$ & $0.2954(14)$ & $0.4732(7)$ & $0.0173(2)$ & $0.0520(17)$ \\
\hline C7A & $0.603(4)$ & $0.043(3)$ & $0.6369(11)$ & $0.0267(3)$ & $0.0520(17)$ \\
\hline H7D & 0.545542 & 0.110860 & 0.669177 & $0.040^{*}$ & $0.0520(17)$ \\
\hline H7E & 0.508277 & -0.028421 & 0.617575 & $0.040^{*}$ & $0.0520(17)$ \\
\hline $\mathrm{H} 7 \mathrm{~F}$ & 0.706943 & -0.011442 & 0.661987 & $0.040^{*}$ & $0.0520(17)$ \\
\hline $\mathrm{C} 8 \mathrm{~A}$ & $0.783(2)$ & $0.415(3)$ & $0.3584(9)$ & $0.0181(2)$ & $0.0520(17)$ \\
\hline C9A & $0.890(3)$ & $0.487(2)$ & $0.3025(7)$ & $0.0233(3)$ & $0.0520(17)$ \\
\hline $\mathrm{H} 9 \mathrm{C}$ & 1.025101 & 0.495079 & 0.318091 & $0.028^{*}$ & $0.0520(17)$ \\
\hline H9D & 0.876172 & 0.427022 & 0.259173 & $0.028^{*}$ & $0.0520(17)$ \\
\hline N3 & $0.67894(16)$ & $0.63909(15)$ & $0.24804(6)$ & $0.0255(3)$ & $0.9480(17)$ \\
\hline N4 & $0.65868(16)$ & $0.79091(15)$ & $0.24611(6)$ & $0.0252(3)$ & $0.9480(17)$ \\
\hline $\mathrm{H} 4$ & $0.574(3)$ & $0.834(3)$ & $0.2145(10)$ & $0.036(6)^{*}$ & $0.9480(17)$ \\
\hline $\mathrm{C} 10$ & $0.81137(15)$ & 0.61509 (16) & $0.30054(6)$ & $0.0208(2)$ & $0.9480(17)$ \\
\hline C11 & $0.87425(16)$ & $0.75120(17)$ & $0.33163(7)$ & $0.0218(2)$ & $0.9480(17)$ \\
\hline H11 & 0.966736 & 0.764031 & 0.369572 & $0.026^{*}$ & $0.9480(17)$ \\
\hline $\mathrm{C} 12$ & $0.77310(17)$ & $0.86140(17)$ & $0.29529(6)$ & $0.0219(3)$ & $0.9480(17)$ \\
\hline $\mathrm{C} 13$ & $0.7758(2)$ & $1.0282(2)$ & $0.30308(9)$ & $0.0301(3)$ & $0.9480(17)$ \\
\hline H13A & 0.898183 & 1.059745 & 0.325150 & $0.045^{*}$ & $0.9480(17)$ \\
\hline H13B & 0.675980 & 1.059071 & 0.332152 & $0.045^{*}$ & $0.9480(17)$ \\
\hline $\mathrm{H} 13 \mathrm{C}$ & 0.754972 & 1.074905 & 0.257033 & $0.045^{*}$ & $0.9480(17)$ \\
\hline N3A & $0.6378(14)$ & $0.668(2)$ & $0.2606(3)$ & $0.0255(3)$ & $0.0520(17)$ \\
\hline $\mathrm{N} 4 \mathrm{~A}$ & $0.6309(16)$ & $0.819(2)$ & $0.2618(3)$ & $0.0252(3)$ & $0.0520(17)$ \\
\hline $\mathrm{H} 4 \mathrm{~B}$ & 0.530526 & 0.868604 & 0.244696 & $0.036(6)^{*}$ & $0.0520(17)$ \\
\hline $\mathrm{C} 10 \mathrm{~A}$ & $0.8062(14)$ & $0.639(2)$ & $0.2901(3)$ & $0.0208(2)$ & $0.0520(17)$ \\
\hline $\mathrm{C} 11 \mathrm{~A}$ & $0.900(2)$ & $0.773(2)$ & $0.3088(5)$ & $0.0218(2)$ & $0.0520(17)$ \\
\hline H11B & 1.023731 & 0.780410 & 0.330902 & $0.026^{*}$ & $0.0520(17)$ \\
\hline $\mathrm{C} 12 \mathrm{~A}$ & $0.783(3)$ & $0.891(2)$ & $0.2898(6)$ & $0.0219(3)$ & $0.0520(17)$ \\
\hline $\mathrm{C} 13 \mathrm{~A}$ & $0.809(4)$ & $1.053(3)$ & $0.2971(9)$ & $0.0301(3)$ & $0.0520(17)$ \\
\hline H13D & 0.934950 & 1.073535 & 0.319707 & $0.045^{*}$ & $0.0520(17)$ \\
\hline $\mathrm{H} 13 \mathrm{E}$ & 0.797247 & 1.099750 & 0.250883 & $0.045^{*}$ & $0.0520(17)$ \\
\hline $\mathrm{H} 13 \mathrm{~F}$ & 0.713733 & 1.094159 & 0.325523 & $0.045^{*}$ & $0.0520(17)$ \\
\hline
\end{tabular}

Atomic displacement parameters $\left(\AA^{2}\right)$

\begin{tabular}{lllllll}
\hline & $U^{11}$ & $U^{22}$ & $U^{33}$ & $U^{12}$ & $U^{13}$ & $U^{23}$ \\
\hline O1 & $0.0175(4)$ & $0.0273(6)$ & $0.0240(6)$ & $-0.0023(4)$ & $-0.0030(4)$ & $0.0038(5)$ \\
N1 & $0.0196(5)$ & $0.0157(5)$ & $0.0292(6)$ & $0.0003(4)$ & $-0.0008(4)$ & $0.0004(4)$ \\
N2 & $0.0152(4)$ & $0.0210(5)$ & $0.0216(5)$ & $-0.0027(4)$ & $-0.0012(4)$ & $0.0056(4)$
\end{tabular}




\begin{tabular}{|c|c|c|c|c|c|c|}
\hline O1A & $0.0175(4)$ & $0.0273(6)$ & $0.0240(6)$ & $-0.0023(4)$ & -0.0030 & $0.0038(5)$ \\
\hline N1A & $0.0196(5)$ & $0.0157(5)$ & $0.0292(6)$ & $0.0003(4)$ & -0.0008 & $0.0004(4)$ \\
\hline $\mathrm{N} 2 \mathrm{~A}$ & $0.0152(4)$ & $0.0210(5)$ & $0.0216(5)$ & $-0.0027(4)$ & $-0.0012(4)$ & $0.0056(4)$ \\
\hline $\mathrm{C} 1$ & $0.0140(5)$ & $0.0165(5)$ & $0.0237(6)$ & $-0.0001(4)$ & $-0.0026(4)$ & $0.0018(4)$ \\
\hline $\mathrm{C} 2$ & $0.0183(5)$ & $0.0204(6)$ & $0.0245(6)$ & $0.0016(4)$ & $0.0020(4)$ & $0.0013(5)$ \\
\hline $\mathrm{C} 3$ & $0.0192(5)$ & $0.0217(6)$ & $0.0244(6)$ & -0.0005 & $0.0029(4)$ & $0.0049(5)$ \\
\hline $\mathrm{C} 4$ & $0.0185(5)$ & $0.0165(5)$ & $0.0250(6)$ & $-0.0017(4)$ & -0.0003 & $0.0042(4)$ \\
\hline $\mathrm{C} 5$ & $0.0184(5)$ & $0.0170(5)$ & $0.0211(5)$ & $-0.0004(4)$ & -0.0003 & $0.0012(4)$ \\
\hline C6 & $0.0153(5)$ & $0.0163(5)$ & $0.0200(5)$ & $-0.0016(4)$ & -0.0012 & $0.0032(4)$ \\
\hline $\mathrm{C} 7$ & $0.0276(6)$ & $0.0175(6)$ & $0.0350(7)$ & $-0.0023(5)$ & $0.0027(5)$ & $0.0061(5)$ \\
\hline $\mathrm{C} 8$ & $0.0183(5)$ & $0.0153(5)$ & $0.0204(6)$ & $0.0008(4)$ & $0.0000(4)$ & $0.0021(4)$ \\
\hline C9 & $0.0233(6)$ & $0.0237(6)$ & $0.0233(6)$ & $0.0026(5)$ & $0.0052(5)$ & $0.0059(5)$ \\
\hline $\mathrm{C} 1 \mathrm{~A}$ & $0.0140(5)$ & $0.0165(5)$ & $0.0237(6)$ & $-0.0001(4)$ & $-0.0026(4)$ & $0.0018(4)$ \\
\hline $\mathrm{C} 2 \mathrm{~A}$ & $0.0183(5)$ & $0.0204(6)$ & $0.0245(6)$ & $0.0016(4)$ & $0.0020(4)$ & $0.0013(5)$ \\
\hline $\mathrm{C} 3 \mathrm{~A}$ & $0.0192(5)$ & $0.0217(6)$ & $0.0244(6)$ & -0.0005 & $0.0029(4)$ & $0.0049(5)$ \\
\hline $\mathrm{C} 4 \mathrm{~A}$ & $0.0185(5)$ & $0.0165(5)$ & $0.0250(6)$ & $-0.0017(4)$ & -0.0003 & $0.0042(4)$ \\
\hline $\mathrm{C} 5 \mathrm{~A}$ & $0.0184(5)$ & $0.0170(5)$ & $0.0211(5)$ & $-0.0004(4)$ & -0.0003 & $0.0012(4)$ \\
\hline C6A & $0.0153(5)$ & $0.0163(5)$ & $0.0200(5)$ & $-0.0016(4)$ & -0.0012 & $0.0032(4)$ \\
\hline C7A & $0.0276(6)$ & $0.0175(6)$ & $0.0350(7)$ & $-0.0023(5)$ & $0.0027(5)$ & $0.0061(5)$ \\
\hline C8A & $0.0183(5)$ & $0.0153(5)$ & $0.0204(6)$ & 0.0008 & $0.0000(4)$ & $0.0021(4)$ \\
\hline C9A & $0.0233(6)$ & $0.0237(6)$ & $0.0233(6)$ & $0.0026(5)$ & $0.0052(5)$ & $0.0059(5)$ \\
\hline N3 & $0.0249(6)$ & $0.0251(6)$ & $0.0254(5)$ & 0.0008 (4) & -0.0042 & $0.0001(4)$ \\
\hline N4 & $0.0234(5)$ & $0.0266(6)$ & $0.0244(6)$ & $0.0024(4)$ & -0.0054 & $0.0024(5)$ \\
\hline $\mathrm{C} 10$ & $0.0180(5)$ & $0.0231(6)$ & $0.0210(5)$ & 0.0005 (4) & 0.0003 & 0.0049 (4) \\
\hline $\mathrm{C} 11$ & $0.0175(5)$ & $0.0257(6)$ & $0.0213(6)$ & $0.0004(4)$ & $-0.0027(4)$ & $0.0026(5)$ \\
\hline $\mathrm{C} 12$ & $0.0179(5)$ & $0.0232(7)$ & $0.0243(6)$ & $0.0012(5)$ & 0.0005 & $0.0033(5)$ \\
\hline $\mathrm{C} 13$ & $0.0283(8)$ & $0.0251(7)$ & $0.0368(8)$ & $0.0005(6)$ & $0.0012(6)$ & $0.0012(6)$ \\
\hline $\mathrm{N} 3 \mathrm{~A}$ & $0.0249(6)$ & $0.0251(6)$ & $0.0254(5)$ & 0.0008 & -0.0042 & $0.0001(4)$ \\
\hline $\mathrm{N} 4 \mathrm{~A}$ & $0.0234(5)$ & $0.0266(6)$ & $0.0244(6)$ & 0.0024 & -0.0054 & $0.0024(5)$ \\
\hline C10A & $0.0180(5)$ & $0.0231(6)$ & $0.0210(5)$ & 0.0005 & 0.0003 & $0.0049(4)$ \\
\hline C11A & $0.0175(5)$ & $0.0257(6)$ & $0.0213(6)$ & 0.0004 & -0.0027 & $0.0026(5)$ \\
\hline $\mathrm{C} 12 \mathrm{~A}$ & $0.0179(5)$ & $0.0232(7)$ & $0.0243(6)$ & $0.0012(5)$ & 0.0005 & $0.0033(5)$ \\
\hline $\mathrm{C} 13 \mathrm{~A}$ & $0.0283(8)$ & $0.0251(7)$ & $0.0368(8)$ & $0.0005(6)$ & $0.0012(6)$ & $0.0012(6)$ \\
\hline
\end{tabular}

Geometric parameters $\left(\AA,{ }^{\circ}\right)$

\begin{tabular}{llll}
\hline O1-C8 & $1.2376(16)$ & C3A-C4A & 1.3900 \\
N1-C1 & $1.4112(17)$ & C3A-C7A & $1.508(3)$ \\
N1-H1A & 0.9099 & C4A-C5A & 1.3900 \\
N1-H1B & 0.9099 & C4A-H4A & 0.9500 \\
N2-C8 & $1.3471(17)$ & C5A-C6A & 1.3900 \\
N2-C6 & $1.4347(17)$ & C5A-H5A & 0.9500 \\
N2-H2A & 0.9100 & C7A-H7D & 0.9800 \\
O1A-C8A & $1.238(3)$ & C7A-H7E & 0.9800 \\
N1A-C1A & $1.412(3)$ & C7A-H7F & 0.9800 \\
N1A-H1C & 0.9099 & C8A-C9A & $1.511(3)$ \\
N1A-H1D & 0.9100 & C9A-C10A & $1.496(3)$ \\
N2A-C8A & $1.347(3)$ & C9A-H9C & 0.9900
\end{tabular}




\begin{tabular}{|c|c|c|c|}
\hline $\mathrm{N} 2 \mathrm{~A}-\mathrm{C} 6 \mathrm{~A}$ & $1.434(3)$ & C9A-H9D & 0.9900 \\
\hline $\mathrm{N} 2 \mathrm{~A}-\mathrm{H} 2 \mathrm{C}$ & 0.8800 & $\mathrm{~N} 3-\mathrm{C} 10$ & $1.3425(18)$ \\
\hline $\mathrm{C} 1-\mathrm{C} 6$ & $1.3995(19)$ & $\mathrm{N} 3-\mathrm{N} 4$ & $1.3635(19)$ \\
\hline $\mathrm{C} 1-\mathrm{C} 2$ & $1.4022(19)$ & $\mathrm{N} 4-\mathrm{C} 12$ & $1.3534(19)$ \\
\hline $\mathrm{C} 2-\mathrm{C} 3$ & $1.388(2)$ & $\mathrm{N} 4-\mathrm{H} 4$ & $0.907(9)$ \\
\hline $\mathrm{C} 2-\mathrm{H} 2$ & 0.9500 & $\mathrm{C} 10-\mathrm{C} 11$ & $1.412(2)$ \\
\hline $\mathrm{C} 3-\mathrm{C} 4$ & $1.397(2)$ & $\mathrm{C} 11-\mathrm{C} 12$ & $1.3766(19)$ \\
\hline $\mathrm{C} 3-\mathrm{H} 3$ & 0.9500 & $\mathrm{C} 11-\mathrm{H} 11$ & 0.9500 \\
\hline $\mathrm{C} 4-\mathrm{C} 5$ & $1.3925(18)$ & $\mathrm{C} 12-\mathrm{C} 13$ & $1.497(2)$ \\
\hline $\mathrm{C} 4-\mathrm{C} 7$ & $1.5077(19)$ & $\mathrm{C} 13-\mathrm{H} 13 \mathrm{~A}$ & 0.9800 \\
\hline $\mathrm{C} 5-\mathrm{C} 6$ & $1.3956(18)$ & $\mathrm{C} 13-\mathrm{H} 13 \mathrm{~B}$ & 0.9800 \\
\hline $\mathrm{C} 5-\mathrm{H} 5$ & 0.9500 & $\mathrm{C} 13-\mathrm{H} 13 \mathrm{C}$ & 0.9800 \\
\hline $\mathrm{C} 7-\mathrm{H} 7 \mathrm{~A}$ & 0.9800 & $\mathrm{~N} 3 \mathrm{~A}-\mathrm{C} 10 \mathrm{~A}$ & $1.311(12)$ \\
\hline C7-H7B & 0.9800 & $\mathrm{~N} 3 \mathrm{~A}-\mathrm{N} 4 \mathrm{~A}$ & $1.351(17)$ \\
\hline $\mathrm{C} 7-\mathrm{H} 7 \mathrm{C}$ & 0.9800 & $\mathrm{~N} 4 \mathrm{~A}-\mathrm{C} 12 \mathrm{~A}$ & $1.331(16)$ \\
\hline $\mathrm{C} 8-\mathrm{C} 9$ & $1.5119(18)$ & $\mathrm{N} 4 \mathrm{~A}-\mathrm{H} 4 \mathrm{~B}$ & 0.8800 \\
\hline $\mathrm{C} 9-\mathrm{C} 10$ & $1.496(2)$ & $\mathrm{C} 10 \mathrm{~A}-\mathrm{C} 11 \mathrm{~A}$ & $1.397(16)$ \\
\hline C9-H9A & 0.9900 & $\mathrm{C} 11 \mathrm{~A}-\mathrm{C} 12 \mathrm{~A}$ & $1.375(17)$ \\
\hline C9-H9B & 0.9900 & $\mathrm{C} 11 \mathrm{~A}-\mathrm{H} 11 \mathrm{~B}$ & 0.9500 \\
\hline $\mathrm{C} 1 \mathrm{~A}-\mathrm{C} 2 \mathrm{~A}$ & 1.3900 & $\mathrm{C} 12 \mathrm{~A}-\mathrm{C} 13 \mathrm{~A}$ & $1.468(17)$ \\
\hline $\mathrm{C} 1 \mathrm{~A}-\mathrm{C} 6 \mathrm{~A}$ & 1.3900 & $\mathrm{C} 13 \mathrm{~A}-\mathrm{H} 13 \mathrm{D}$ & 0.9800 \\
\hline $\mathrm{C} 2 \mathrm{~A}-\mathrm{C} 3 \mathrm{~A}$ & 1.3900 & $\mathrm{C} 13 \mathrm{~A}-\mathrm{H} 13 \mathrm{E}$ & 0.9800 \\
\hline $\mathrm{C} 2 \mathrm{~A}-\mathrm{H} 2 \mathrm{~B}$ & 0.9500 & $\mathrm{C} 13 \mathrm{~A}-\mathrm{H} 13 \mathrm{~F}$ & 0.9800 \\
\hline $\mathrm{C} 1-\mathrm{N} 1-\mathrm{H} 1 \mathrm{~A}$ & 113.0 & $\mathrm{C} 6 \mathrm{~A}-\mathrm{C} 5 \mathrm{~A}-\mathrm{H} 5 \mathrm{~A}$ & 120.0 \\
\hline $\mathrm{C} 1-\mathrm{N} 1-\mathrm{H} 1 \mathrm{~B}$ & 107.5 & $\mathrm{C} 4 \mathrm{~A}-\mathrm{C} 5 \mathrm{~A}-\mathrm{H} 5 \mathrm{~A}$ & 120.0 \\
\hline $\mathrm{H} 1 \mathrm{~A}-\mathrm{N} 1-\mathrm{H} 1 \mathrm{~B}$ & 111.9 & $\mathrm{C} 5 \mathrm{~A}-\mathrm{C} 6 \mathrm{~A}-\mathrm{C} 1 \mathrm{~A}$ & 120.0 \\
\hline $\mathrm{C} 8-\mathrm{N} 2-\mathrm{C} 6$ & $121.97(11)$ & $\mathrm{C} 5 \mathrm{~A}-\mathrm{C} 6 \mathrm{~A}-\mathrm{N} 2 \mathrm{~A}$ & $120.1(13)$ \\
\hline $\mathrm{C} 8-\mathrm{N} 2-\mathrm{H} 2 \mathrm{~A}$ & 117.4 & $\mathrm{C} 1 \mathrm{~A}-\mathrm{C} 6 \mathrm{~A}-\mathrm{N} 2 \mathrm{~A}$ & $119.7(13)$ \\
\hline $\mathrm{C} 6-\mathrm{N} 2-\mathrm{H} 2 \mathrm{~A}$ & 120.6 & $\mathrm{C} 3 \mathrm{~A}-\mathrm{C} 7 \mathrm{~A}-\mathrm{H} 7 \mathrm{D}$ & 109.5 \\
\hline $\mathrm{C} 1 \mathrm{~A}-\mathrm{N} 1 \mathrm{~A}-\mathrm{H} 1 \mathrm{C}$ & 110.0 & $\mathrm{C} 3 \mathrm{~A}-\mathrm{C} 7 \mathrm{~A}-\mathrm{H} 7 \mathrm{E}$ & 109.5 \\
\hline $\mathrm{C} 1 \mathrm{~A}-\mathrm{N} 1 \mathrm{~A}-\mathrm{H} 1 \mathrm{D}$ & 113.5 & $\mathrm{H} 7 \mathrm{D}-\mathrm{C} 7 \mathrm{~A}-\mathrm{H} 7 \mathrm{E}$ & 109.5 \\
\hline $\mathrm{H} 1 \mathrm{C}-\mathrm{N} 1 \mathrm{~A}-\mathrm{H} 1 \mathrm{D}$ & 111.9 & $\mathrm{C} 3 \mathrm{~A}-\mathrm{C} 7 \mathrm{~A}-\mathrm{H} 7 \mathrm{~F}$ & 109.5 \\
\hline $\mathrm{C} 8 \mathrm{~A}-\mathrm{N} 2 \mathrm{~A}-\mathrm{C} 6 \mathrm{~A}$ & $123.6(15)$ & $\mathrm{H} 7 \mathrm{D}-\mathrm{C} 7 \mathrm{~A}-\mathrm{H} 7 \mathrm{~F}$ & 109.5 \\
\hline $\mathrm{C} 8 \mathrm{~A}-\mathrm{N} 2 \mathrm{~A}-\mathrm{H} 2 \mathrm{C}$ & 118.2 & $\mathrm{H} 7 \mathrm{E}-\mathrm{C} 7 \mathrm{~A}-\mathrm{H} 7 \mathrm{~F}$ & 109.5 \\
\hline $\mathrm{C} 6 \mathrm{~A}-\mathrm{N} 2 \mathrm{~A}-\mathrm{H} 2 \mathrm{C}$ & 118.2 & $\mathrm{O} 1 \mathrm{~A}-\mathrm{C} 8 \mathrm{~A}-\mathrm{N} 2 \mathrm{~A}$ & $120.4(18)$ \\
\hline $\mathrm{C} 6-\mathrm{C} 1-\mathrm{C} 2$ & $118.12(12)$ & $\mathrm{O} 1 \mathrm{~A}-\mathrm{C} 8 \mathrm{~A}-\mathrm{C} 9 \mathrm{~A}$ & $126(2)$ \\
\hline $\mathrm{C} 6-\mathrm{C} 1-\mathrm{N} 1$ & $120.68(12)$ & $\mathrm{N} 2 \mathrm{~A}-\mathrm{C} 8 \mathrm{~A}-\mathrm{C} 9 \mathrm{~A}$ & $113.7(11)$ \\
\hline $\mathrm{C} 2-\mathrm{C} 1-\mathrm{N} 1$ & $121.12(12)$ & $\mathrm{C} 10 \mathrm{~A}-\mathrm{C} 9 \mathrm{~A}-\mathrm{C} 8 \mathrm{~A}$ & $106.6(14)$ \\
\hline $\mathrm{C} 3-\mathrm{C} 2-\mathrm{C} 1$ & $120.77(13)$ & $\mathrm{C} 10 \mathrm{~A}-\mathrm{C} 9 \mathrm{~A}-\mathrm{H} 9 \mathrm{C}$ & 110.4 \\
\hline $\mathrm{C} 3-\mathrm{C} 2-\mathrm{H} 2$ & 119.6 & $\mathrm{C} 8 \mathrm{~A}-\mathrm{C} 9 \mathrm{~A}-\mathrm{H} 9 \mathrm{C}$ & 110.4 \\
\hline $\mathrm{C} 1-\mathrm{C} 2-\mathrm{H} 2$ & 119.6 & $\mathrm{C} 10 \mathrm{~A}-\mathrm{C} 9 \mathrm{~A}-\mathrm{H} 9 \mathrm{D}$ & 110.4 \\
\hline $\mathrm{C} 2-\mathrm{C} 3-\mathrm{C} 4$ & $121.24(13)$ & $\mathrm{C} 8 \mathrm{~A}-\mathrm{C} 9 \mathrm{~A}-\mathrm{H} 9 \mathrm{D}$ & 110.4 \\
\hline $\mathrm{C} 2-\mathrm{C} 3-\mathrm{H} 3$ & 119.4 & $\mathrm{H} 9 \mathrm{C}-\mathrm{C} 9 \mathrm{~A}-\mathrm{H} 9 \mathrm{D}$ & 108.6 \\
\hline $\mathrm{C} 4-\mathrm{C} 3-\mathrm{H} 3$ & 119.4 & $\mathrm{C} 10-\mathrm{N} 3-\mathrm{N} 4$ & $104.22(12)$ \\
\hline $\mathrm{C} 5-\mathrm{C} 4-\mathrm{C} 3$ & $118.03(12)$ & $\mathrm{C} 12-\mathrm{N} 4-\mathrm{N} 3$ & $112.76(12)$ \\
\hline $\mathrm{C} 5-\mathrm{C} 4-\mathrm{C} 7$ & $120.82(13)$ & $\mathrm{C} 12-\mathrm{N} 4-\mathrm{H} 4$ & $126.7(16)$ \\
\hline $\mathrm{C} 3-\mathrm{C} 4-\mathrm{C} 7$ & $121.13(13)$ & $\mathrm{N} 3-\mathrm{N} 4-\mathrm{H} 4$ & $120.5(16)$ \\
\hline
\end{tabular}




\begin{tabular}{|c|c|}
\hline $\mathrm{C} 4-\mathrm{C} 5-\mathrm{C} 6$ & $121.19(12)$ \\
\hline $\mathrm{C} 4-\mathrm{C} 5-\mathrm{H} 5$ & 119.4 \\
\hline $\mathrm{C} 6-\mathrm{C} 5-\mathrm{H} 5$ & 119.4 \\
\hline $\mathrm{C} 5-\mathrm{C} 6-\mathrm{C} 1$ & $120.64(12)$ \\
\hline $\mathrm{C} 5-\mathrm{C} 6-\mathrm{N} 2$ & $119.46(12)$ \\
\hline $\mathrm{C} 1-\mathrm{C} 6-\mathrm{N} 2$ & $119.89(11)$ \\
\hline $\mathrm{C} 4-\mathrm{C} 7-\mathrm{H} 7 \mathrm{~A}$ & 109.5 \\
\hline $\mathrm{C} 4-\mathrm{C} 7-\mathrm{H} 7 \mathrm{~B}$ & 109.5 \\
\hline $\mathrm{H} 7 \mathrm{~A}-\mathrm{C} 7-\mathrm{H} 7 \mathrm{~B}$ & 109.5 \\
\hline $\mathrm{C} 4-\mathrm{C} 7-\mathrm{H} 7 \mathrm{C}$ & 109.5 \\
\hline $\mathrm{H} 7 \mathrm{~A}-\mathrm{C} 7-\mathrm{H} 7 \mathrm{C}$ & 109.5 \\
\hline $\mathrm{H} 7 \mathrm{~B}-\mathrm{C} 7-\mathrm{H} 7 \mathrm{C}$ & 109.5 \\
\hline $\mathrm{O} 1-\mathrm{C} 8-\mathrm{N} 2$ & $122.20(12)$ \\
\hline $\mathrm{O} 1-\mathrm{C} 8-\mathrm{C} 9$ & $121.58(12)$ \\
\hline $\mathrm{N} 2-\mathrm{C} 8-\mathrm{C} 9$ & $116.22(11)$ \\
\hline $\mathrm{C} 10-\mathrm{C} 9-\mathrm{C} 8$ & $111.99(9)$ \\
\hline $\mathrm{C} 10-\mathrm{C} 9-\mathrm{H} 9 \mathrm{~A}$ & 109.2 \\
\hline $\mathrm{C} 8-\mathrm{C} 9-\mathrm{H} 9 \mathrm{~A}$ & 109.2 \\
\hline $\mathrm{C} 10-\mathrm{C} 9-\mathrm{H} 9 \mathrm{~B}$ & 109.2 \\
\hline $\mathrm{C} 8-\mathrm{C} 9-\mathrm{H} 9 \mathrm{~B}$ & 109.2 \\
\hline $\mathrm{H} 9 \mathrm{~A}-\mathrm{C} 9-\mathrm{H} 9 \mathrm{~B}$ & 107.9 \\
\hline $\mathrm{C} 2 \mathrm{~A}-\mathrm{C} 1 \mathrm{~A}-\mathrm{C} 6 \mathrm{~A}$ & 120.0 \\
\hline $\mathrm{C} 2 \mathrm{~A}-\mathrm{C} 1 \mathrm{~A}-\mathrm{N} 1 \mathrm{~A}$ & $119.99(8)$ \\
\hline $\mathrm{C} 6 \mathrm{~A}-\mathrm{C} 1 \mathrm{~A}-\mathrm{N} 1 \mathrm{~A}$ & $120.01(8)$ \\
\hline $\mathrm{C} 1 \mathrm{~A}-\mathrm{C} 2 \mathrm{~A}-\mathrm{C} 3 \mathrm{~A}$ & 120.0 \\
\hline $\mathrm{C} 1 \mathrm{~A}-\mathrm{C} 2 \mathrm{~A}-\mathrm{H} 2 \mathrm{~B}$ & 120.0 \\
\hline $\mathrm{C} 3 \mathrm{~A}-\mathrm{C} 2 \mathrm{~A}-\mathrm{H} 2 \mathrm{~B}$ & 120.0 \\
\hline $\mathrm{C} 4 \mathrm{~A}-\mathrm{C} 3 \mathrm{~A}-\mathrm{C} 2 \mathrm{~A}$ & 120.0 \\
\hline $\mathrm{C} 4 \mathrm{~A}-\mathrm{C} 3 \mathrm{~A}-\mathrm{C} 7 \mathrm{~A}$ & $119.3(14)$ \\
\hline $\mathrm{C} 2 \mathrm{~A}-\mathrm{C} 3 \mathrm{~A}-\mathrm{C} 7 \mathrm{~A}$ & $120.6(14)$ \\
\hline $\mathrm{C} 3 \mathrm{~A}-\mathrm{C} 4 \mathrm{~A}-\mathrm{C} 5 \mathrm{~A}$ & 120.0 \\
\hline $\mathrm{C} 3 \mathrm{~A}-\mathrm{C} 4 \mathrm{~A}-\mathrm{H} 4 \mathrm{~A}$ & 120.0 \\
\hline $\mathrm{C} 5 \mathrm{~A}-\mathrm{C} 4 \mathrm{~A}-\mathrm{H} 4 \mathrm{~A}$ & 120.0 \\
\hline $\mathrm{C} 6 \mathrm{~A}-\mathrm{C} 5 \mathrm{~A}-\mathrm{C} 4 \mathrm{~A}$ & 120.0 \\
\hline $\mathrm{C} 6-\mathrm{C} 1-\mathrm{C} 2-\mathrm{C} 3$ & $-0.18(19)$ \\
\hline $\mathrm{N} 1-\mathrm{C} 1-\mathrm{C} 2-\mathrm{C} 3$ & $-177.18(12)$ \\
\hline $\mathrm{C} 1-\mathrm{C} 2-\mathrm{C} 3-\mathrm{C} 4$ & $-0.5(2)$ \\
\hline $\mathrm{C} 2-\mathrm{C} 3-\mathrm{C} 4-\mathrm{C} 5$ & $0.6(2)$ \\
\hline $\mathrm{C} 2-\mathrm{C} 3-\mathrm{C} 4-\mathrm{C} 7$ & $-178.08(13)$ \\
\hline $\mathrm{C} 3-\mathrm{C} 4-\mathrm{C} 5-\mathrm{C} 6$ & $-0.09(19)$ \\
\hline $\mathrm{C} 7-\mathrm{C} 4-\mathrm{C} 5-\mathrm{C} 6$ & $178.64(13)$ \\
\hline $\mathrm{C} 4-\mathrm{C} 5-\mathrm{C} 6-\mathrm{C} 1$ & $-0.60(19)$ \\
\hline $\mathrm{C} 4-\mathrm{C} 5-\mathrm{C} 6-\mathrm{N} 2$ & $-179.60(11)$ \\
\hline $\mathrm{C} 2-\mathrm{C} 1-\mathrm{C} 6-\mathrm{C} 5$ & $0.73(18)$ \\
\hline $\mathrm{N} 1-\mathrm{C} 1-\mathrm{C} 6-\mathrm{C} 5$ & $177.74(12)$ \\
\hline $\mathrm{C} 2-\mathrm{C} 1-\mathrm{C} 6-\mathrm{N} 2$ & $179.72(11)$ \\
\hline $\mathrm{N} 1-\mathrm{C} 1-\mathrm{C} 6-$ & \\
\hline
\end{tabular}

N3-C10-C11 111.17 (12)

N3-C10-C9 $119.95(13)$

$\mathrm{C} 11-\mathrm{C} 10-\mathrm{C} 9 \quad 128.88(12)$

$\mathrm{C} 12-\mathrm{C} 11-\mathrm{C} 10 \quad 105.37$ (12)

$\mathrm{C} 12-\mathrm{C} 11-\mathrm{H} 11$

$\mathrm{C} 10-\mathrm{C} 11-\mathrm{H} 11$

$\mathrm{N} 4-\mathrm{C} 12-\mathrm{C} 11$

$\mathrm{N} 4-\mathrm{C} 12-\mathrm{C} 13 \quad 122.33(14)$

$\mathrm{C} 11-\mathrm{C} 12-\mathrm{C} 13 \quad 131.18(14)$

$\mathrm{C} 12-\mathrm{C} 13-\mathrm{H} 13 \mathrm{~A} \quad 109.5$

$\mathrm{C} 12-\mathrm{C} 13-\mathrm{H} 13 \mathrm{~B} \quad 109.5$

$\mathrm{H} 13 \mathrm{~A}-\mathrm{C} 13-\mathrm{H} 13 \mathrm{~B} \quad 109.5$

$\mathrm{C} 12-\mathrm{C} 13-\mathrm{H} 13 \mathrm{C} \quad 109.5$

$\mathrm{H} 13 \mathrm{~A}-\mathrm{C} 13-\mathrm{H} 13 \mathrm{C} \quad 109.5$

$\mathrm{H} 13 \mathrm{~B}-\mathrm{C} 13-\mathrm{H} 13 \mathrm{C} \quad 109.5$

$\mathrm{C} 10 \mathrm{~A}-\mathrm{N} 3 \mathrm{~A}-\mathrm{N} 4 \mathrm{~A} \quad 102.8(12)$

$\mathrm{C} 12 \mathrm{~A}-\mathrm{N} 4 \mathrm{~A}-\mathrm{N} 3 \mathrm{~A} \quad 117.0(13)$

$\mathrm{C} 12 \mathrm{~A}-\mathrm{N} 4 \mathrm{~A}-\mathrm{H} 4 \mathrm{~B} \quad 121.5$

N3A-N4A-H4B 121.5

$\mathrm{N} 3 \mathrm{~A}-\mathrm{C} 10 \mathrm{~A}-\mathrm{C} 11 \mathrm{~A} \quad 110.3(14)$

N3A-C10A-C9A $125.8(16)$

$\mathrm{C} 11 \mathrm{~A}-\mathrm{C} 10 \mathrm{~A}-\mathrm{C} 9 \mathrm{~A} \quad 123.9(14)$

$\mathrm{C} 12 \mathrm{~A}-\mathrm{C} 11 \mathrm{~A}-\mathrm{C} 10 \mathrm{~A} \quad 108.4$ (13)

$\mathrm{C} 12 \mathrm{~A}-\mathrm{C} 11 \mathrm{~A}-\mathrm{H} 11 \mathrm{~B} \quad 125.8$

$\mathrm{C} 10 \mathrm{~A}-\mathrm{C} 11 \mathrm{~A}-\mathrm{H} 11 \mathrm{~B} \quad 125.8$

$\mathrm{N} 4 \mathrm{~A}-\mathrm{C} 12 \mathrm{~A}-\mathrm{C} 11 \mathrm{~A} \quad 101.4(13)$

$\mathrm{N} 4 \mathrm{~A}-\mathrm{C} 12 \mathrm{~A}-\mathrm{C} 13 \mathrm{~A} \quad 127.2(19)$

$\mathrm{C} 11 \mathrm{~A}-\mathrm{C} 12 \mathrm{~A}-\mathrm{C} 13 \mathrm{~A} \quad 131.3(18)$

$\mathrm{C} 12 \mathrm{~A}-\mathrm{C} 13 \mathrm{~A}-\mathrm{H} 13 \mathrm{D} \quad 109.5$

$\mathrm{C} 12 \mathrm{~A}-\mathrm{C} 13 \mathrm{~A}-\mathrm{H} 13 \mathrm{E} \quad 109.5$

H13D-C13A-H13E $\quad 109.5$

C12A-C13A-H13F $\quad 109.5$

$\mathrm{H} 13 \mathrm{D}-\mathrm{C} 13 \mathrm{~A}-\mathrm{H} 13 \mathrm{~F} \quad 109.5$

$\mathrm{H} 13 \mathrm{E}-\mathrm{C} 13 \mathrm{~A}-\mathrm{H} 13 \mathrm{~F} \quad 109.5$

$\mathrm{C} 2 \mathrm{~A}-\mathrm{C} 1 \mathrm{~A}-\mathrm{C} 6 \mathrm{~A}-\mathrm{N} 2 \mathrm{~A} \quad 175.7$ (15)

$\mathrm{N} 1 \mathrm{~A}-\mathrm{C} 1 \mathrm{~A}-\mathrm{C} 6 \mathrm{~A}-\mathrm{N} 2 \mathrm{~A} \quad-4.3(15)$

$\mathrm{C} 8 \mathrm{~A}-\mathrm{N} 2 \mathrm{~A}-\mathrm{C} 6 \mathrm{~A}-\mathrm{C} 5 \mathrm{~A} \quad 90(3)$

$\mathrm{C} 8 \mathrm{~A}-\mathrm{N} 2 \mathrm{~A}-\mathrm{C} 6 \mathrm{~A}-\mathrm{C} 1 \mathrm{~A} \quad-86(3)$

$\mathrm{C} 6 \mathrm{~A}-\mathrm{N} 2 \mathrm{~A}-\mathrm{C} 8 \mathrm{~A}-\mathrm{O} 1 \mathrm{~A} \quad 9(5)$

$\mathrm{C} 6 \mathrm{~A}-\mathrm{N} 2 \mathrm{~A}-\mathrm{C} 8 \mathrm{~A}-\mathrm{C} 9 \mathrm{~A} \quad-173$ (2)

$\mathrm{O} 1 \mathrm{~A}-\mathrm{C} 8 \mathrm{~A}-\mathrm{C} 9 \mathrm{~A}-\mathrm{C} 10 \mathrm{~A} \quad 59$ (4)

$\mathrm{N} 2 \mathrm{~A}-\mathrm{C} 8 \mathrm{~A}-\mathrm{C} 9 \mathrm{~A}-\mathrm{C} 10 \mathrm{~A}-119$ (2)

$\mathrm{C} 10-\mathrm{N} 3-\mathrm{N} 4-\mathrm{C} 12 \quad-0.05(3)$

$\mathrm{N} 4-\mathrm{N} 3-\mathrm{C} 10-\mathrm{C} 11 \quad 0.05$ (2)

N4-N3- C10-C9 -179.92 (3)

$\mathrm{C} 8-\mathrm{C} 9-\mathrm{C} 10-\mathrm{N} 3 \quad-95.38$ (12)

$\mathrm{C} 8-\mathrm{C} 9-\mathrm{C} 10-\mathrm{C} 11$ 


$\begin{array}{ll}\mathrm{C} 8-\mathrm{N} 2-\mathrm{C} 6-\mathrm{C} 5 & 90.79(16) \\ \mathrm{C} 8-\mathrm{N} 2-\mathrm{C} 6-\mathrm{C} 1 & -88.21(16) \\ \mathrm{C} 6-\mathrm{N} 2-\mathrm{C} 8-\mathrm{O} 1 & 4.1(2) \\ \mathrm{C} 6-\mathrm{N} 2-\mathrm{C} 8-\mathrm{C} 9 & -176.27(12) \\ \mathrm{O} 1-\mathrm{C} 8-\mathrm{C} 9-\mathrm{C} 10 & 64.49(19) \\ \mathrm{N} 2-\mathrm{C} 8-\mathrm{C} 9-\mathrm{C} 10 & -115.16(14) \\ \mathrm{C} 6 \mathrm{~A}-\mathrm{C} 1 \mathrm{~A}-\mathrm{C} 2 \mathrm{~A}-\mathrm{C} 3 \mathrm{~A} & 0.0 \\ \mathrm{~N} 1 \mathrm{~A}-\mathrm{C} 1 \mathrm{~A}-\mathrm{C} 2 \mathrm{~A}-\mathrm{C} 3 \mathrm{~A} & 180.0 \\ \mathrm{C} 1 \mathrm{~A}-\mathrm{C} 2 \mathrm{~A}-\mathrm{C} 3 \mathrm{~A}-\mathrm{C} 4 \mathrm{~A} & 0.0 \\ \mathrm{C} 1 \mathrm{~A}-\mathrm{C} 2 \mathrm{~A}-\mathrm{C} 3 \mathrm{~A}-\mathrm{C} 7 \mathrm{~A} & 177.6(19) \\ \mathrm{C} 2 \mathrm{~A}-\mathrm{C} 3 \mathrm{~A}-\mathrm{C} 4 \mathrm{~A}-\mathrm{C} 5 \mathrm{~A} & 0.0 \\ \mathrm{C} 7 \mathrm{~A}-\mathrm{C} 3 \mathrm{~A}-\mathrm{C} 4 \mathrm{~A}-\mathrm{C} 5 \mathrm{~A} & -177.6(18) \\ \mathrm{C} 3 \mathrm{~A}-\mathrm{C} 4 \mathrm{~A}-\mathrm{C} 5 \mathrm{~A}-\mathrm{C} 6 \mathrm{~A} & 0.0 \\ \mathrm{C} 4 \mathrm{~A}-\mathrm{C} 5 \mathrm{~A}-\mathrm{C} 6 \mathrm{~A}-\mathrm{C} 1 \mathrm{~A} & 0.0 \\ \mathrm{C} 4 \mathrm{~A}-\mathrm{C} 5 \mathrm{~A}-\mathrm{C} 6 \mathrm{~A}-\mathrm{N} 2 \mathrm{~A} & -175.7(15) \\ \mathrm{C} 2 \mathrm{~A}-\mathrm{C} 1 \mathrm{~A}-\mathrm{C} 6 \mathrm{~A}-\mathrm{C} 5 \mathrm{~A} & 0.0 \\ \mathrm{~N} 1 \mathrm{~A}-\mathrm{C} 1 \mathrm{~A}-\mathrm{C} 6 \mathrm{~A}-\mathrm{C} 5 \mathrm{~A} & 180.0\end{array}$

$\begin{array}{ll}\mathrm{N} 3-\mathrm{C} 10-\mathrm{C} 11-\mathrm{C} 12 & -0.04(4) \\ \mathrm{C} 9-\mathrm{C} 10-\mathrm{C} 11-\mathrm{C} 12 & 179.93(5) \\ \mathrm{N} 3-\mathrm{N} 4-\mathrm{C} 12-\mathrm{C} 11 & 0.02(5) \\ \mathrm{N} 3-\mathrm{N} 4-\mathrm{C} 12-\mathrm{C} 13 & 179.89(4) \\ \mathrm{C} 10-\mathrm{C} 11-\mathrm{C} 12-\mathrm{N} 4 & 0.01(5) \\ \mathrm{C} 10-\mathrm{C} 11-\mathrm{C} 12-\mathrm{C} 13 & -179.84(5) \\ \mathrm{C} 10 \mathrm{~A}-\mathrm{N} 3 \mathrm{~A}-\mathrm{N} 4 \mathrm{~A}-\mathrm{C} 12 \mathrm{~A} & 0.00(3) \\ \mathrm{N} 4 \mathrm{~A}-\mathrm{N} 3 \mathrm{~A}-\mathrm{C} 10 \mathrm{~A}-\mathrm{C} 11 \mathrm{~A} & 0.00(3) \\ \mathrm{N} 4 \mathrm{~A}-\mathrm{N} 3 \mathrm{~A}-\mathrm{C} 10 \mathrm{~A}-\mathrm{C} 9 \mathrm{~A} & 180.00(4) \\ \mathrm{C} 8 \mathrm{~A}-\mathrm{C} 9 \mathrm{~A}-\mathrm{C} 10 \mathrm{~A}-\mathrm{N} 3 \mathrm{~A} & -69.6(15) \\ \mathrm{C} 8 \mathrm{~A}-\mathrm{C} 9 \mathrm{~A}-\mathrm{C} 10 \mathrm{~A}-\mathrm{C} 11 \mathrm{~A} & 110.4(15) \\ \mathrm{N} 3 \mathrm{~A}-\mathrm{C} 10 \mathrm{~A}-\mathrm{C} 11 \mathrm{~A}-\mathrm{C} 12 \mathrm{~A} & 0.00(5) \\ \mathrm{C} 9 \mathrm{~A}-\mathrm{C} 10 \mathrm{~A}-\mathrm{C} 11 \mathrm{~A}-\mathrm{C} 12 \mathrm{~A} & 179.99(6) \\ \mathrm{N} 3 \mathrm{~A}-\mathrm{N} 4 \mathrm{~A}-\mathrm{C} 12 \mathrm{~A}-\mathrm{C} 11 \mathrm{~A} & 0.00(5) \\ \mathrm{N} 3 \mathrm{~A}-\mathrm{N} 4 \mathrm{~A}-\mathrm{C} 12 \mathrm{~A}-\mathrm{C} 13 \mathrm{~A} & 180.00(5) \\ \mathrm{C} 10 \mathrm{~A}-\mathrm{C} 11 \mathrm{~A}-\mathrm{C} 12 \mathrm{~A}-\mathrm{N} 4 \mathrm{~A} & 0.00(6) \\ \mathrm{C} 10 \mathrm{~A}-\mathrm{C} 11 \mathrm{~A}-\mathrm{C} 12 \mathrm{~A}-\mathrm{C} 13 \mathrm{~A} & 180.00(6)\end{array}$

Hydrogen-bond geometry $\left(\AA,{ }^{\circ}\right)$

\begin{tabular}{lllll}
\hline$D-\mathrm{H} \cdots A$ & $D-\mathrm{H}$ & $\mathrm{H} \cdots A$ & $D \cdots A$ & $D-\mathrm{H} \cdots A$ \\
\hline $\mathrm{N} 1-\mathrm{H} 1 B \cdots \mathrm{O} 1^{\mathrm{i}}$ & 0.91 & 2.13 & $3.0284(19)$ & 171 \\
$\mathrm{~N} 2-\mathrm{H} 2 A \cdots \mathrm{N} 1^{\mathrm{ii}}$ & 0.91 & 2.14 & $3.0354(17)$ & 170 \\
$\mathrm{C} 2-\mathrm{H} 2 \cdots \mathrm{O} 1^{\mathrm{i}}$ & 0.95 & 2.62 & $3.334(2)$ & 132 \\
$\mathrm{~N} 4-\mathrm{H} 4 \cdots \mathrm{O} 1^{\mathrm{iii}}$ & $0.91(1)$ & $1.99(1)$ & $2.8625(17)$ & $163(2)$ \\
\hline
\end{tabular}

Symmetry codes: (i) $-x+1,-y+1,-z+1$; (ii) $-x+2,-y+1,-z+1$; (iii) $-x+1, y+1 / 2,-z+1 / 2$. 